\title{
ECONOMICS
}

\section{FUTURE ECONOMISTS AND THE ROLE OF THE PhD CONFERENCE}

by

Kenneth W Clements

Economics Discipline, Business School, University of Western Australia

and

Robert G Gregory

Australian National University 


\title{
FUTURE ECONOMISTS AND THE ROLE OF THE PhD CONFERENCE*
}

by

\author{
Kenneth W Clements \\ UWA Business School \\ and \\ Robert G Gregory
}

ANU

\begin{abstract}
$\underline{\text { Abstract }}$
The PhD Conference in Economics and Business was a unique Australian innovation when it commenced in the late 1980s. With the high-quality feedback from discussants, the conference was a productive special event, even horizon-broadening for some students. The conference is now a partnership between six universities and has involved almost 900 students from many universities. This paper places the conference in a broader context and highlights some of the stars by identifying 54 student-presenters now full professors.
\end{abstract}

\footnotetext{
${ }^{*}$ Thanks to Denzil Fiebig, Izan and Mei Han for helpful comments and discussions. For excellent research assistance, we thank Mohammad Farhad, Tom Simpson and Vuong Vu.
} 


\section{The Origins}

In the 1980s both of us were enthusiastic supervisors of PhD students -- we wanted to see the students do well, promote their work and wanted to expand our activities in this area. A major stumbling block was that the students were only exposed to us and as good as we were (and still are!), we felt the need for them to interact more with the wider profession to have the benefit of critical comments from others, to get to know others and to feel part of the vibrant international industry carrying out research in economics. This type of interaction would help students appreciate the value of economics, the importance of a research-friendly culture and the value of having supportive, like-minded colleagues at other institutions, etc., all of which contributes to creative research over the longer term. Leading universities in the other countries devote substantial resources to helping their PhDs along these lines, as we had experienced ourselves at the LSE, Northwestern, Chicago and elsewhere. A wholesale transplant of the North American model was, of course, always out of the question and never an objective, but we did have firm ideas about how the training of Australian economics PhDs could be enhanced. It should be emphasised that other colleagues at UWA and ANU shared these views and contributed substantially to the PhD conferences, as will be discussed.

The first PhD Conference was held at UWA in 1987, and for several subsequent years, it was held there annually (except for 1990 when we ran out of money!). Interestingly, the "isolated" location of UWA in Perth seemed to help the conferences. The time taken to travel to Perth (5 or so hours from Canberra) tended to make it easier for visitors to commit to the whole conference, rather than coming in for one day, or even only for the one session they were involved with. Additionally, for a number of participants from other parts of Australia, the conference might have been the opportunity to visit the beautiful City of Perth for the first time. Consequently, there was a nice quorum at most sessions which assisted in generating productive discussion, controversy and feedback for the students.

ANU was strongly represented by presenters and discussants at the early conferences and became a partner with UWA, with the location alternating between Perth and Canberra. This provided a deeper national focus and a sharing of the administrative burden of convening an annual conference. The ANU hosted the conference in 1992 for the first time, and the ANU-UWA partnership has continued to work in a remarkably effective way ever since. One reason for the enduring relationship is the flexibility of the arrangements that have remained informal with nothing written down and no involvement of the bureaucracy of either university. Another key ingredient is the warm personal relationship between the two 
of us (this may come as a surprise to those who seem to believe we are "professionally incompatible”).

In later years, four other universities joined the partnership. Paul Frijters arranged for the conference to be held at the University of Queensland for the first time in 2011; David Harris, Stephen King and Pushkar Maitra were the organisers at Monash in 2014; Nisvan Erkal at the University of Melbourne in 2017; and Gigi Foster at UNSW in 2018. Then, in 2019, the conference returned to ANU, where it was organised by Bob Breunig. In 2020, the conference will have an on-line format due to the pandemic and be hosted by Monash with Pushkar Maitra as the leader of a team of organisers. All these individuals have done outstanding jobs and contributed substantially to the strong reputation of the conference series. This growth in the partnership of organising universities has been organic and unsolicited, and has further enhanced opportunities for students.

\section{The Model}

Each conference involves about $30 \mathrm{PhD}$ students presenting papers on their inprogress research and the same number of discussants. Economics departments are invited to nominate students to present. Students have come from many Australian and New Zealand universities, as well as some from leading foreign institutions.

The role of the discussants is most important to the success of the conferences. Discussants comment on the strengths and weaknesses of the assigned paper and to produce something like an extensive referee report for a high-quality journal. Discussants are asked to be critical but constructive and engage with students. There is a delicate balance between building the self-confidence of young scholars, while at the same time preparing them for the rigours of professional life by pointing to improvements that can be made to their work. Good discussants think seriously how best to strike this balance, and tend to adopt a friendly but direct tone in their comments.

Peer pressure has the clear effect of stimulating discussants to excel, to think deeply about the issues and how the paper might be extended and/or improved. This has led to exceptional contributions by discussants that have been immensely beneficial to students. More than once, prior to the conference, a discussant has asked the student for his/her data and completely redid the analysis. On another occasion, a discussant identified a crucial error in the paper -- and the student was forever grateful to be able to rectify the problem before the thesis was examined. We have been lucky to have a number of impressive discussants such as Denzil Fiebig, Gigi Foster, Paul Frijters and Geoff Kingston. 
It would not be unusual for a discussant to devote something like a week to an assigned paper. Why are these busy people, who face so many demands, willing to invest so much time? Most are simply public-spirited in wanting to help students. There is also the peer pressure mentioned above, as well as the prestige of being part of a high-quality team. Additionally, self-interest is at stake as there is a prize for the best discussant awarded on the basis of a popular vote by discussants and students. A rough estimate of the implicit value of the prize can be obtained with a difference-in-difference analysis of citations. As Marc Blaug says, "citations are the coinage of reward in academia”. As compared to discussants who did not win the prize, the annual number of citations to the work of winners increases by, on average, 56 , or by about $27 \%$. This increase occurs in each subsequent year after the prize. There may be an issue about attributing all the additional cites to the prize, but it does point to its approximate value.

\section{Links with Accounting and Finance}

There are many commonalities between economics and finance. Many of the same research approaches are used, the same professional language is used, and many of the same students are taught by those in economics and accounting/finance. In this sense, the separation of departments of economics and finance at most Australian universities seems a bit strange. Izan, then Professor of Accounting and Finance at UWA (and wife of Clements) had the good idea of bringing the two groups together by organising a stream at the conference devoted to accounting and finance. This has been highly successful in helping both students and discussants become more familiar with others outside their immediate area, and to share ideas. A number of participants have commented on the value of this almost unique opportunity to interact.

The accounting and finance stream is run when the conference is held at UWA, and at the 2018 conference held at the University of Melbourne. There is considerable scope for further developing this productive cross-disciplinary link at some of the other partner universities.

\section{$\underline{\text { Resources }}$}

The resources required to stage the conferences are substantial. A major cost is the opportunity cost of professional time that has been so generously provided by individuals and institutions. There is also travel and accommodation of discussants, the cost of which is higher when the conference is held in Perth. The students' home universities fund their travel and accommodation costs. 
In addition to the partner universities, the costs have in large part been funded by external sponsors. These include major employers of economists, companies, individuals and professional bodies with an interest in research in economics and finance and/or those who are simply happy to support a good cause involving promising young scholars. Especially valuable has been the longer-term sponsorship of the Reserve Bank of Australia, the Productivity Commission, the Economic Society of Australia and its WA Branch, the Treasury and the Australian Bureau of Statistics. Without this generous support, the conferences would not have been possible.

\section{Professors-To-Be}

The conference plays a talent-spotting function. As many $\mathrm{PhD}$ students aspire to careers in academia, we have carried out a preliminary investigation of those who become a full professor (level E academic) at an Australian university, or the equivalent. While promotion and hiring practices differ across institutions and fluctuate over time, perhaps 25 percent of all academics in economics and finance are professors. Focusing on professors of course misses all those who have embarked on successful academic careers without becoming a professor. Our only justification is one of manageability: With almost 900 students, obtaining an accurate picture of all the subsequent careers is a daunting task we do not wish to undertake. ${ }^{1} \mathrm{~A}$ number of PhDs are now leaders in their careers outside of academia, but we have not attempted any systematic tracking of those.

In what follows, we consider those who presented at the 16 conferences held between 1987 and 2003. This allows for a substantial period between presenting at the conference and achieving the rank of professor; this ranges from up to (i) 33 years for those presenting in 1987, and (ii) 17 years for 2003 presenters. The 2003 cut-off is not inconsistent with the average age at the appointment as a professor which must be above 40 . The disadvantage of this approach is that it misses the now-professors who presented post-2003 (such as Peter Siminski at UTS, who presented in 2007, and David Johnson at Monash, in 2005), as well as stars who are yet a professor (such as Tim Kam at ANU, 2002). These considerations point to the lower-bound nature of the following findings.

Between 1987 and 2003 there were 411 presentations by students. In the early years, two students presented twice -- Jacqui Dwyer and David Gruen, who, interestingly, both have gone on to stellar careers outside academia, Dwyer as a senior RBA official and Gruen as Australian Statistician at the ABS. Thus, there were 409 individuals involved. Table 1 reveals

\footnotetext{
${ }^{1}$ Again for reasons of manageability, those who have become a professor outside of Australia have been mostly excluded.
} 
54 of these presenters became professors, or $\frac{54}{409}=13$ percent. An interpretation might be there is a 13 percent probability of becoming a professor (other things equal), reasonable odds for ambitious young scholars. As our approach may have missed some professors-to-be, this is probably a lower bound.

Table 2 gives the distribution of the years in which the now-professors presented. There is a tendency for some bunching in the earlier years, possibly reflecting a pent-up supply. The numbers tend to drop off at the end of the period, which is reasonable given the usual time taken to achieve the professorial rank. From an average conference, 3.4 presenters become professors. Table 3 shows that the now-professors are located at a number of Australia's top universities. The ANU is the most enthusiastic employer, followed by UQ, Melbourne and Monash. Interestingly, this pattern matches fairly closely the well-known rankings of universities. It is noteworthy that a little over one-half of the 54 are now professors at Group-of-Eight (Go8) universities. ${ }^{2}$

Where did these professors did get the $\mathrm{PhD}$ and does this matter for the subsequent destination? To shed some light on this, we cross-classify the 54 professors according to where they came from and where they are now located, as in panel A of Table 4. This shows that 41 were trained at a Go8 university and 24 subsequently returned to such a university as a professor. Interestingly, the Go8 hired only 4 trained at a non-Go8 institution. The non-Go8 hired 26 of the 54, and $\frac{17}{26}=65$ percent came from the Go8. Several individual Go8 universities are identified separately in panel B of the table. ANU dominates as a both a producer and consumer of the professors -- of the 54, 14 were trained at the ANU and 7 are now professors at Go8 universities, including 4 back at ANU. The case of ANU and Melbourne is an interesting one: As a combination, they have hired 11 of the 54, and none were trained at a non-Go8 institution.

It is good to see so many locally trained PhDs becoming professors. But is there a problem with the hiring practices of the Go8? Why do they hire so many of their own? Is it just that the Go8 tend to attract better $\mathrm{PhD}$ students and/or provide better training, or is there a cultural barrier? In many cases, after closely observing a person's work for, say, 4 years, a good deal of information will have accumulated locally, information more difficult for

\footnotetext{
${ }^{2}$ How representative are these results? On average, Australian universities produce about $100 \mathrm{PhDs}$ in economics p. a. (Clements and Si, 2019). Each conference has about 30 students, or about 30 percent of the total for an average year. There is the usual variability of quality of the papers presented, but the conferences tend to attract the stronger students. Thus, the sample is possibly skewed towards those who will ultimately become a professor. This would have the effect of pushing upwards the estimated probably of becoming a professor and partially offsets the above factors making for the lower-bound interpretation of the results.
} 
outsiders to learn about. This may account for the Go8 hiring Go8 students. There is, of course, the not-so-innocent reason that the practice is incestuous that blocks the inflow of talented outsiders.

More generally, the above results indicate a past willingness, or even an enthusiasm, for Australian universities to hire Australian-trained PhDs. In recent times the situation seems to have changed markedly, however, with a growing trend to appoint people from overseas. Those with Australian degrees now seem to find it more difficult to land academic jobs in Australia, especially with the Go8.

\section{Reflections}

The PhD Conference series has involved almost 900 PhD students from 48 different universities and has been embraced by almost all Australian universities, major employers of economists and other government, professional and business organisations. The conferences have led to the productive sharing of ideas, research collaboration and job opportunities for students. At least two partnerships formed at the conference have led to marriage, and no known divorces!

Each year, there is a range of students, some world-beaters, others who struggle a bit, but all are highly motivated and keen to learn and succeed. The topics of the papers tend to come and go, perhaps reflecting broader trends (fashions?) in economics. There is now less emphasis on international economics and labour economics, more on applied econometrics with panel data sets. There seems to be a perennial under-supply of macro papers - maybe the controversy and disputation makes a macro thesis too risky. The majority of students now come from overseas, reflecting the composition of many PhD programs in Australia and elsewhere. This may account for the few papers dealing with Australian economic issues. There is a recent tendency for papers presented to be jointly authored with supervisors, which also seems to reflect a broader trend. This is an undesirable development that makes it more difficult to judge the capabilities and promise of the students, not their co-authors. One aspect that did not take off was the idea of developing a local job market at the conference. It did for the best students, of course, but not a general market.

Now there are many conferences and funding available for students to participate, but back in the late 1980s this was absent. This emphasises the important innovation of the conference, but also indicate that it is perhaps not as important as it once was. More than once, the conference has been described by others as "the best in Australia". A former presenter now a prominent professor, wrote saying attending the conference was a "life-long experience”. Another sign of success is that the model has been emulated by others, other 
universities and other disciplines. The conferences have attracted one Nobel Laureate (Robert Mundell) and who knows how many Nobels-to-be!

As in comedy, timing is everything in economics conferences. The timing was right for our conference, which coincided with several important developments. First and foremost, the last 30 years have been boom times for economics and finance (even if it didn't feel it at the time). There have been record numbers of students at all university levels, a flourishing of research and an associated increase in funding. Second, in the 1990s and beyond there was the wave of coursework PhDs in Australia and the need for PhD students to move on from narrowly defined English-style $\mathrm{PhD}$ books. This was associated with growth in the influence of US-type training, a productive development in our view. Third, there was an increase in graduate programs outside the big institutions - in a certain way, the conference was a way in for smaller universities. Related to these reasons is the rise of empirical/applied work in economics and finance, strongly reflected in the nature of theses. Very few theses are now just theory, even applied theory. Most are what used to be called applied econometrics.

The Appendix gives details of the 32 conferences with summary information on the students involved, discussants, sponsors and so on. A spreadsheet with the underlying data is available on request. Further information is available in the publications listed below.

\section{$\underline{\text { References }}$}

Clements, K. W. (2010). “The PhD Conference in Economics and Business Two Decades On.” Economic Papers 29: 169-80.

Clements, K. W., and D. Chenhall (1995). "The Production of PhDs in Economics and Business by Australian Universities.” Economic Papers 14: 49-66.

Clements, K. W., R. G. Gregory and T. Takayama, eds. (1991). International Economics Postgraduate Research Conference Volume, Supplement to the Economic Record.

Clements, K. W., and Y. Qiang (1999). “Ten Years of the PhD Conference in Economics and Business.” Economic Record 75: 301-12.

Clements, K. W., and J. Si (2019). “What do Australian Economics PhDs Do?” Australian Economic Review 52: 134-44.

Clements, K. W., and P. Wang (2003), “Who Cites What?” Economic Record 73: 229-44. 
Table 1

PRESENTERS NOW PROFESSORS

1987-2003

\begin{tabular}{|c|c|c|}
\hline Presenter & Current Position & $\begin{array}{c}\text { Year of } \\
\text { Conference } \\
\text { Presentation } \\
\end{array}$ \\
\hline 1. Adams, Philip & Professor, Vic Univ, Melbourne & 1987 \\
\hline 2. Bandara, Jayatillek & Professor, Griffith & 1987 \\
\hline 3. Bateman, Hazel & Professor, UNSW & 1994 \\
\hline 4. Brooks, Robert & Professor, Monash & 1991 \\
\hline 5. Bugeja, Martin & Professor, UTS & 2001 \\
\hline 6. Butler, David & Professor, Griffith & 1995 \\
\hline 7. Chand, Satish & Professor, UNSW & 1994 \\
\hline 8. Coxhead, Ian & Professor, Univ of Wisconsin-Madison & 1987 \\
\hline 9. Da Silva Rosa, Ray & Professor, UWA & 1991 \\
\hline 10. Daly, Anne & Emeritus Professor, Univ of Canberra & 1989 \\
\hline 11. Daly, Kevin & Professor, UWS & 1993 \\
\hline 12. Davidson, Sinclair & Professor, RMIT & 1996 \\
\hline 13. Dennis, Richard & Univ of Glasgow & 1999 \\
\hline 14. Dungey, Mardi & Formerly Professor, Univ of Tasmania & 1995 \\
\hline 15. Dwyer, Jacqui & Head, Information Department, RBA & 1988, 1991 \\
\hline 16. Ellis, Luci & Assistant Governor, RBA & 2002 \\
\hline 17. Fry-McKibbin, Renée & Professor, ANU & 2001 \\
\hline 18. Gordon, Jenny & Formerly Principal Advisor, Prod Comm & 1987 \\
\hline 19. Gray, Matthew & Professor, ANU & 1997 \\
\hline 20. Gruen, David & Australian Statistician, ABS & 1987, 1988 \\
\hline 21. Heath, Alex & Head, Econ Analysis Department, RBA & 1998 \\
\hline 22. Huang, Yiping & Professor, Peking & 1992 \\
\hline 23. Hunter, Boyd & Professor, ANU & 1993 \\
\hline 24. Jensen, Paul & Professor, Melbourne & 2001 \\
\hline 25. Kalb, Guyonne & Professorial Res Fellow, Melbourne & 1995 \\
\hline 26. Kawaguchi, Akira & Professor, Doshisha Univ & 1989 \\
\hline 27. Liesch, Peter & Professor, UQ & 1987 \\
\hline 28. Martin, Gael & Professor, Monash & 1995 \\
\hline 29. Meagher, Kieron & Professor, ANU & 1996 \\
\hline 30. Meng, Xin & Professor, ANU & 1991 \\
\hline 31. Moore, Greg & Professor, UND & 1992 \\
\hline 32. Moshirian, Fariborz & Professor, UNSW & 1988 \\
\hline 33. Mumford, Karen & Professor, York & 1987 \\
\hline 34. O’Donnell, Chris & Professor, UQ & 1987 \\
\hline 35. Ong, Li Lian & Group Head, ASEAN+3 & 1995 \\
\hline
\end{tabular}


Table 1 (continued)

PRESENTERS NOW PROFESSORS

$1987-2003$

\begin{tabular}{llc}
\hline \hline \multicolumn{1}{c}{ Presenter } & Current Position & $\begin{array}{c}\text { Year of } \\
\text { Conference } \\
\text { Presentation }\end{array}$ \\
\hline 36. Petchey, Jeff & E/Professor, Curtin & 1991 \\
37. Preston, Alison & Professor, UWA & 1995 \\
38. Ralston, Deborah & Professorial Fellow, Monash & 1995 \\
39. Rimmer, Maureen & Professor, Vic Univ, Melbourne & 1989 \\
40. Roca, Eduardo & Professor, Griffith & 1995 \\
41. Romalis, John & Professor, Sydney & 2000 \\
42. Stachurski, John & Professor, ANU & 2000 \\
43. Stonecash, Robin & Professor, S Cross & 1989 \\
44. Strachan, Rodney & Professor, UQ & 1998 \\
45. Tang, KK & Professor, UQ & 1998 \\
46. Thorp, Susan & Professor, Sydney & 2003 \\
47. Tourky, Rabee & Professor, ANU & 1994 \\
48. Twite, Gary & Vising Professor, Melbourne & 1989 \\
49. Vaithianathan, Rhema & Professor, UQ \& Auckland Univ Tech & 1997 \\
50. Wan, Alan & Professor, City Univ of Hong Kong & 1992 \\
51. Wilkins, Roger & Professor, Melbourne & 1999 \\
52. Wittwer, Glyn & Professor, Vic Univ, Melbourne & 1998 \\
53. Wu, Yanrui & Professor, UWA & 1989 \\
54. Zhao, Xueyan & Professor, Monash & 1998 \\
\hline
\end{tabular}

Note: This is an indicative list of those presenters from the first 16 conferences (1987-2003, excluding 1990 when the conference was not held) who are now full professors at an Australian university or (more or less) the equivalent. To identify those who become professors, we used Google in conjunction with three sources of information: (i) Staff lists on web pages of most Australian universities. (ii) Our list of all students who presented. (iii) Our personal knowledge of subsequent careers of presenters. 
Table 2

WHEN DID THE

FUTURE PROFESSORS PRESENT?

\begin{tabular}{lc}
\hline $\begin{array}{c}\text { Year of } \\
\text { Conference }\end{array}$ & $\begin{array}{c}\text { Number of } \\
\text { Professors-To-Be } \\
\text { Presenting }\end{array}$ \\
\hline 1. 1987 & 8 \\
2. 1988 & 2 \\
3. 1989 & 6 \\
4. 1991 & 4 \\
5. 1992 & 3 \\
6. 1993 & 2 \\
7. 1994 & 3 \\
8. 1995 & 8 \\
9. 1996 & 2 \\
10. 1997 & 2 \\
11. 1998 & 5 \\
12. 1999 & 2 \\
13. 2000 & 2 \\
14. 2001 & 3 \\
15. 2002 & 1 \\
16. 2003 & 1 \\
Total & 54 \\
Average & 3.4 \\
\hline
\end{tabular}

Note: This table gives the number of presenters at each conference who are now professors.

Source: Table 1.

Table 3

\begin{tabular}{lc}
\multicolumn{2}{c}{ WHERE ARE THEY NOW? } \\
\hline \hline $\begin{array}{l}\text { Location } \\
\text { of Professors }\end{array}$ & Number \\
\hline ANU & 7 \\
UQ & 5 \\
Melbourne & 4 \\
Monash & 4 \\
Griffith & 3 \\
Victoria & 3 \\
UNSW & 3 \\
UWA & 3 \\
Sydney & 2 \\
Other & 20 \\
Total & 54
\end{tabular}

Note: This table gives the universities at which conference presenters who have become professors are now located. Source: Table 1. 
Table 4

ORIGIN AND DESTINATION OF PROFESSORS

(Number of professors)

A. Go8 vs Other

\begin{tabular}{l||c|c|c|}
\hline \multirow{2}{*}{ Where they are now } & \multicolumn{3}{|c|}{ Where they were a student } \\
\cline { 2 - 4 } & Go8 & Other & Total \\
\hline Go8 & 24 & 4 & 28 \\
\hline Other & 17 & 9 & 26 \\
\hline Total & 41 & 13 & 54 \\
\hline
\end{tabular}

B. Individual Institutions

\begin{tabular}{|c|c|c|c|c|c|c|c|}
\hline \multirow{3}{*}{ Where they are now } & \multicolumn{7}{|c|}{ Where they were a student } \\
\hline & \multicolumn{5}{|c|}{ Go8 } & \multirow{2}{*}{$\begin{array}{l}\text { Non- } \\
\text { Go8 }\end{array}$} & \multirow{2}{*}{ Total } \\
\hline & ANU & UQ & Melbourne & Monash & Other Go8 & & \\
\hline ANU & 4 & 1 & 2 & & & & 7 \\
\hline UQ & 1 & 1 & & 1 & 1 & 1 & 5 \\
\hline Melbourne & & & 1 & 1 & 2 & & 4 \\
\hline Monash & & & & 2 & & 2 & 4 \\
\hline Other Go8 & 1 & & & 1 & 5 & 1 & 8 \\
\hline Other & 8 & 1 & 2 & & 6 & 9 & 26 \\
\hline Total & 14 & 3 & 5 & 5 & 14 & 13 & 54 \\
\hline
\end{tabular}

Source: Tables 1 and A4. 


\section{APPENDIX}

\section{FIGURE A1}

\section{SOURCE OF STUDENTS}

1987-2019

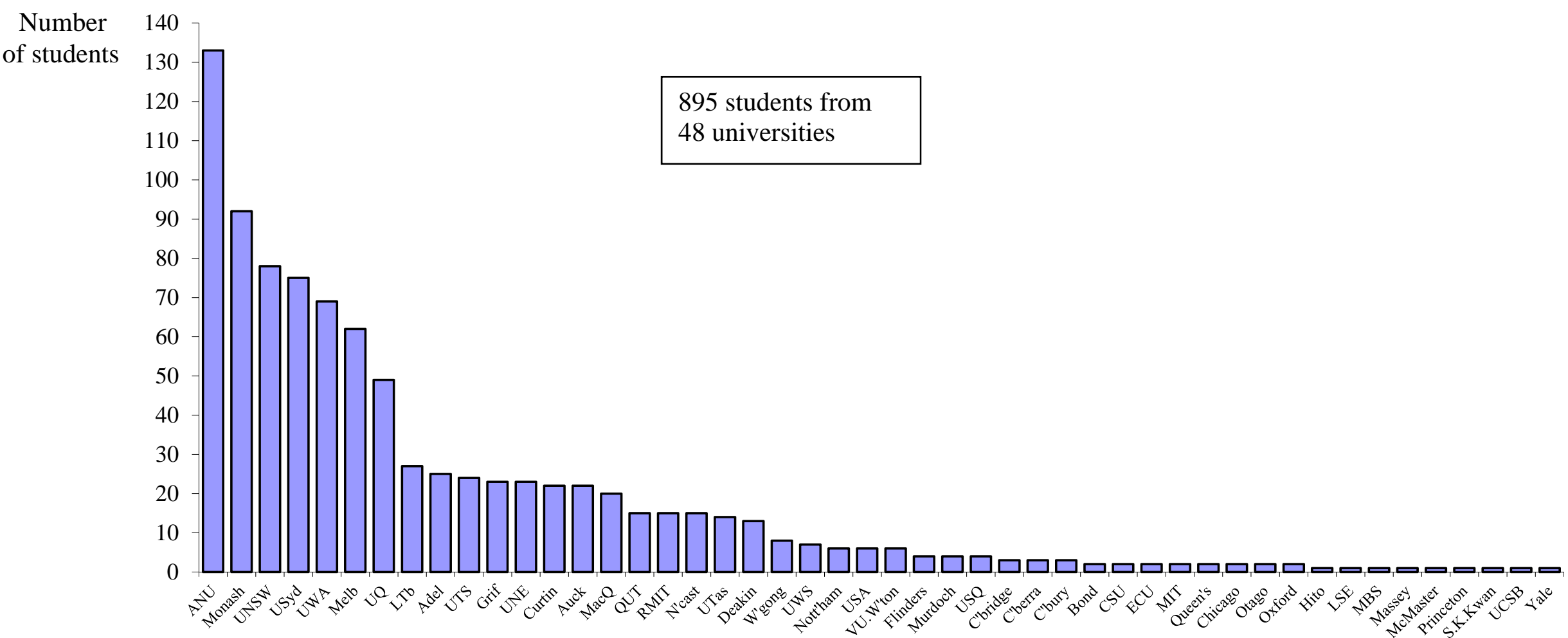

This figure gives the total number of students from each institution that have presented at all the conferences. 


\section{FIGURE A2}

\section{SOURCE OF DISCUSSANTS 1987-2019}

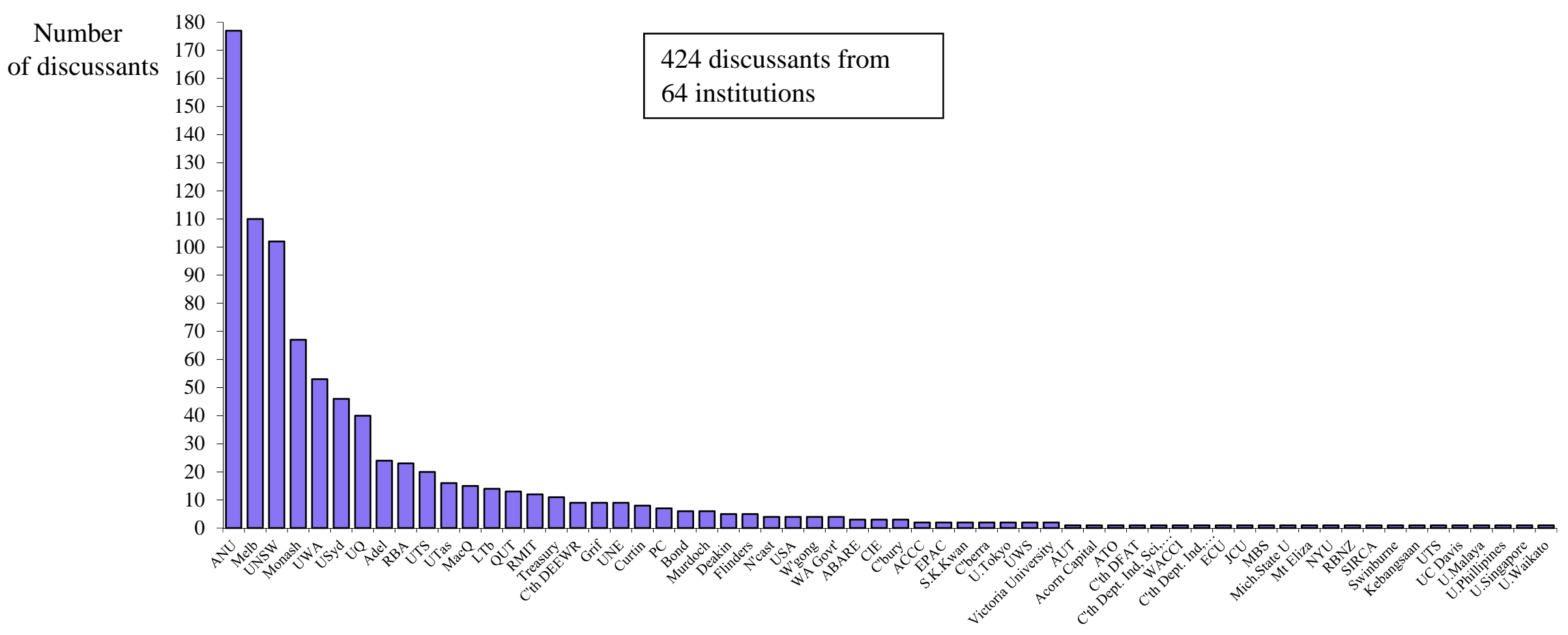

This figure gives the total number of discussants from each institution that have served at all the conferences. 
FIGURE A3

\section{PROMINENT DISCUSSANTS}

1987-2019

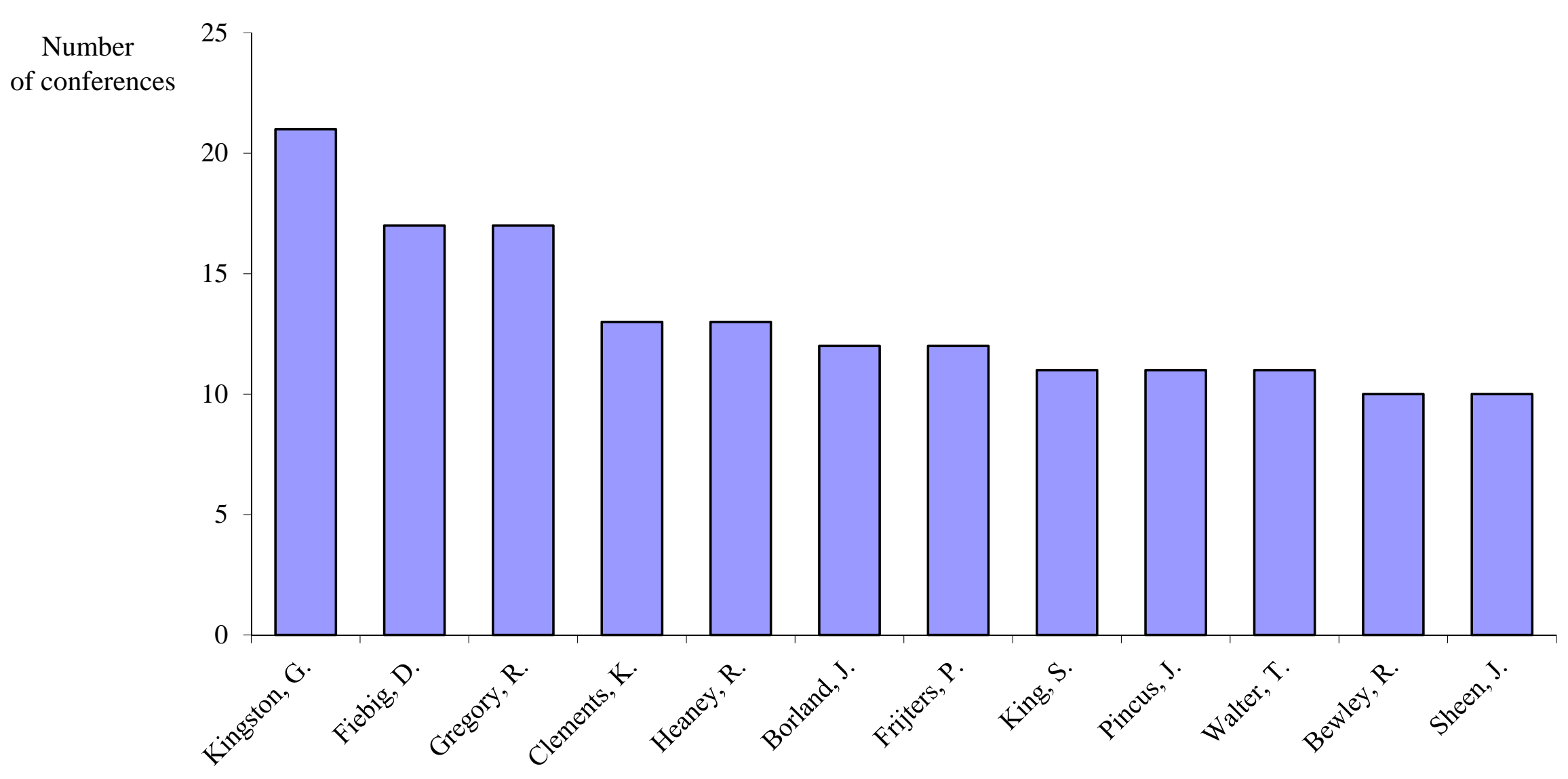

This figure gives the names of individuals who have served a number of times as discussants at the conferences. 
FIGURE A4

FREQUENT SPONSORS

1987-2019

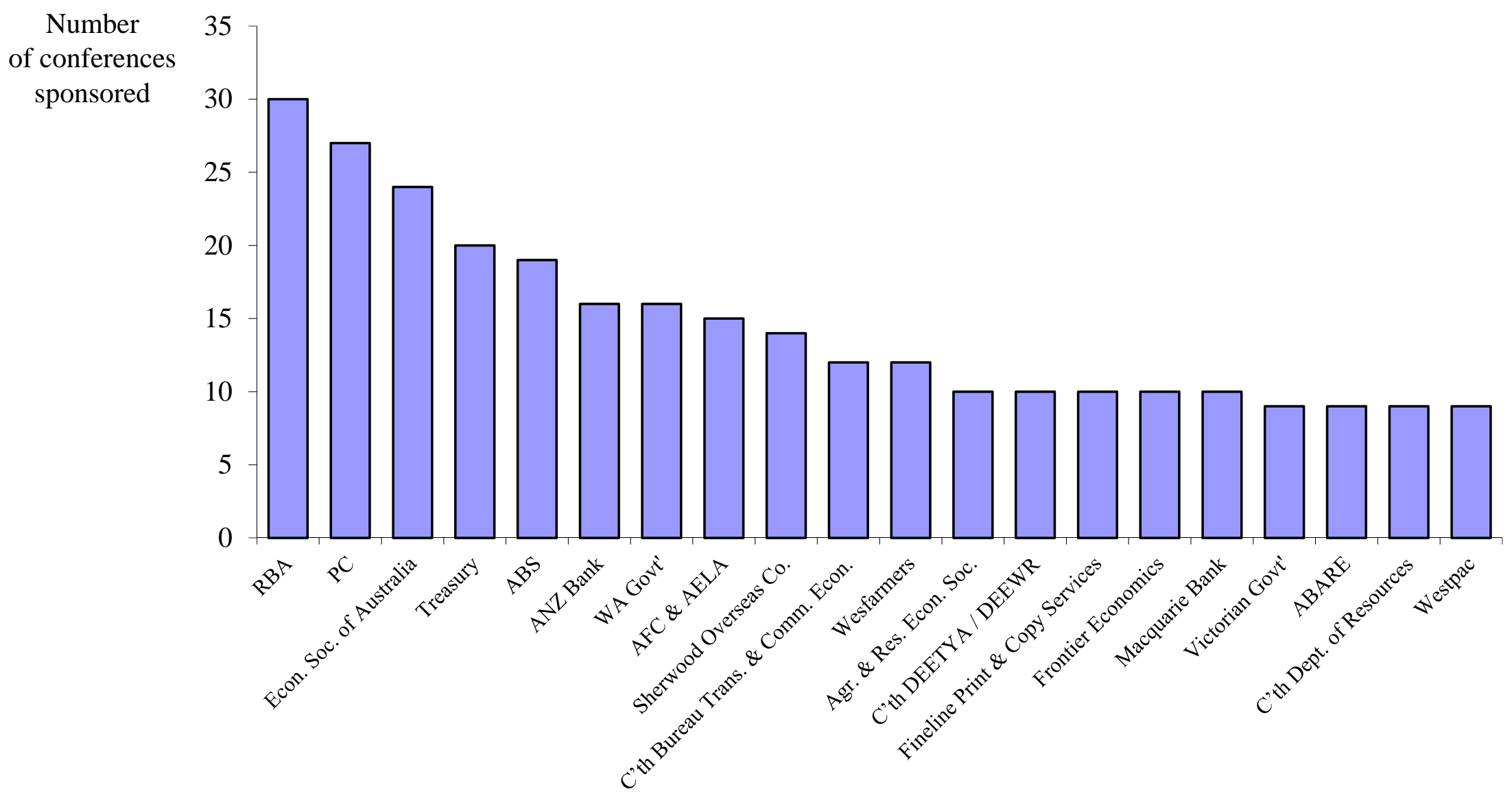

This figure gives the institutions that have sponsored the conference a number of times. 
TABLE A1

DETAILS OF THE CONFERENCES, 1987-2019

\begin{tabular}{|c|c|c|c|c|c|c|c|c|}
\hline Year & Date & $\begin{array}{l}\text { Number } \\
\text { of } \\
\text { students } \\
\text { involved }\end{array}$ & $\begin{array}{l}\text { Number of } \\
\text { discussants } \\
\text { involved }\end{array}$ & $\begin{array}{l}\text { Number of } \\
\text { institutions } \\
\text { involved } \\
\text { (students } \\
\text { and } \\
\text { discussants) }\end{array}$ & Partner institutions & Convenors & Co-ordinators & Location \\
\hline 1987 & Nov. 1-3 & 22 & 17 & 20 & Economic Research Centre, UWA & K. Clements & A. Webber & UWA \\
\hline 1988 & Nov. 6-8 & 15 & 18 & 19 & Economic Research Centre, UWA & K. Clements & A. Webber & UWA \\
\hline 1989 & Nov. 5-7 & 17 & 18 & 17 & Economic Research Centre, UWA & K. Clements & A. Webber & UWA \\
\hline 1991 & Nov. 6-8 & 21 & 21 & 16 & $\begin{array}{l}\text { 1. Economic Research Centre, UWA } \\
\text { 2. Centre for Economic Policy Research, ANU }\end{array}$ & K. Clements & D. Chenhall & UWA \\
\hline 1992 & Nov. 3-5 & 20 & 20 & 18 & $\begin{array}{l}\text { 1. Centre for Economic Policy Research, ANU } \\
\text { 2. Economic Research Centre, UWA }\end{array}$ & A. Pagan & A. Ritchie & ANU \\
\hline 1993 & Nov. 3-5 & 25 & 25 & 21 & $\begin{array}{l}\text { 1. Economic Research Centre, UWA } \\
\text { 2. Research Centre in Accounting and Finance, UWA } \\
\text { 3. Centre for Economic Policy Research, ANU }\end{array}$ & $\begin{array}{l}\text { K. Clements } \\
\text { Izan }\end{array}$ & D. Chenhall & UWA \\
\hline 1994 & Dec. 7-9 & 24 & 24 & 20 & $\begin{array}{l}\text { 1. Centre for Economic Policy Research, ANU } \\
\text { 2. Economic Research Centre, UWA }\end{array}$ & $\begin{array}{l}\text { A. Pagan } \\
\text { S. King }\end{array}$ & R. Carson & ANU \\
\hline 1995 & Nov. 8-10 & 28 & 29 & 17 & $\begin{array}{l}\text { 1. Economic Research Centre, UWA } \\
\text { 2. Research Centre in Accounting and Finance, UWA } \\
\text { 3. Centre for Economic Policy Research, ANU }\end{array}$ & $\begin{array}{l}\text { K. Clements } \\
\text { Izan }\end{array}$ & D. Chenhall & UWA \\
\hline
\end{tabular}


TABLE A1 (continued)

DETAILS OF THE CONFERENCES, 1987-2019

\begin{tabular}{|c|c|c|c|c|c|c|c|c|}
\hline Year & Date & $\begin{array}{l}\text { Number } \\
\text { of } \\
\text { students } \\
\text { involved }\end{array}$ & $\begin{array}{l}\text { Number of } \\
\text { discussants } \\
\text { involved }\end{array}$ & $\begin{array}{l}\text { Number of } \\
\text { institutions } \\
\text { involved } \\
\text { (students } \\
\text { and } \\
\text { discussants) }\end{array}$ & Partner institutions & Convenors & Co-ordinators & Location \\
\hline 1996 & Nov. 7-8 & 23 & 23 & 21 & $\begin{array}{l}\text { 1. Centre for Economic Policy Research, ANU } \\
\text { 2. Economic Research Centre, UWA }\end{array}$ & S. King & R. Carson & ANU \\
\hline 1997 & Nov. 5-7 & 32 & 31 & 23 & $\begin{array}{l}\text { 1. Economic Research Centre, UWA } \\
\text { 2. Centre for Economic Policy Research, ANU } \\
\text { 3. Department of Accounting and Finance, UWA }\end{array}$ & K. Clements & P. Madsen & UWA \\
\hline 1998 & Dec. 7-9 & 24 & 24 & 21 & $\begin{array}{l}\text { 1. Centre for Economic Policy Research, ANU } \\
\text { 2. Economic Research Centre, UWA }\end{array}$ & A. Pagan & R. Carson & ANU \\
\hline 1999 & Nov. $8-10$ & 32 & 33 & 26 & $\begin{array}{l}\text { 1. Economic Research Centre, UWA } \\
\text { 2. Centre for Economic Policy Research, ANU } \\
\text { 3. Department of Accounting and Finance, UWA }\end{array}$ & K. Clements & G. Barrett & UWA \\
\hline 2000 & Nov. 7-8 & 25 & 25 & 19 & $\begin{array}{l}\text { 1. Centre for Economic Policy Research, ANU } \\
\text { 2. Economic Research Centre, UWA }\end{array}$ & B. Chapman & R. Carson & ANU \\
\hline 2001 & Nov. 7-9 & 40 & 39 & 26 & $\begin{array}{l}\text { 1. Economic Research Centre, UWA } \\
\text { 2. Centre for Economic Policy Research, ANU } \\
\text { 3. Graduate School of Management, UWA }\end{array}$ & K. Clements & S. Pollock & UWA \\
\hline 2002 & Nov. 6-8 & 31 & 30 & 20 & $\begin{array}{l}\text { 1. Centre for Economic Policy Research, ANU } \\
\text { 2. Economic Research Centre, UWA }\end{array}$ & $\begin{array}{l}\text { R. Gregory } \\
\text { B. Chapman }\end{array}$ & R. Carson & ANU \\
\hline
\end{tabular}


TABLE A1 (continued)

DETAILS OF THE CONFERENCES, 1987-2019

\begin{tabular}{|c|c|c|c|c|c|c|c|c|}
\hline Year & Date & $\begin{array}{l}\text { Number } \\
\text { of } \\
\text { students } \\
\text { involved }\end{array}$ & $\begin{array}{l}\text { Number of } \\
\text { discussants } \\
\text { involved }\end{array}$ & $\begin{array}{l}\text { Number of } \\
\text { institutions } \\
\text { involved } \\
\text { (students } \\
\text { and } \\
\text { discussants) }\end{array}$ & Partner institutions & Convenors & Co-ordinators & Location \\
\hline 2003 & Nov. 5-7 & 32 & 31 & 22 & $\begin{array}{l}\text { 1. Economic Research Centre, UWA } \\
\text { 2. Centre for Economic Policy Research, ANU }\end{array}$ & $\begin{array}{l}\text { K. Clements } \\
\text { Izan }\end{array}$ & H. Friday & UWA \\
\hline 2004 & Nov. $10-12$ & 25 & 24 & 15 & $\begin{array}{l}\text { 1. Centre for Economic Policy Research, ANU } \\
\text { 2. Economic Research Centre, UWA }\end{array}$ & R. Gregory & L. Elliot & ANU \\
\hline 2005 & Nov. 2-4 & 30 & 29 & 23 & $\begin{array}{l}\text { 1. Economic Research Centre, UWA } \\
\text { 2. Centre for Economic Policy Research, ANU }\end{array}$ & M. McLure & H. Friday & UWA \\
\hline 2006 & Nov. $8-10$ & 27 & 29 & 20 & $\begin{array}{l}\text { 1. Centre for Economic Policy Research, ANU } \\
\text { 2. Economic Research Centre, UWA } \\
\text { 3. Faculty of Business, QUT }\end{array}$ & $\begin{array}{l}\text { R. Gregory } \\
\text { P. Frijters }\end{array}$ & L. Elliot & ANU \\
\hline 2007 & Oct. 31-Nov. 2 & 30 & 30 & 20 & $\begin{array}{l}\text { 1. Business School, UWA } \\
\text { 2. Centre for Economic Policy Research, ANU }\end{array}$ & M. McLure & S. Coakly & UWA \\
\hline 2008 & Nov. 20-21 & 29 & 28 & 20 & $\begin{array}{l}\text { 1. Centre for Economic Policy Research, ANU } \\
\text { 2. Economic Research Centre, UWA } \\
\text { 3. Faculty of Business, QUT }\end{array}$ & $\begin{array}{l}\text { G. Brennan } \\
\text { R. Gregory } \\
\text { P. Frijters }\end{array}$ & $\begin{array}{l}\text { L. Elliot } \\
\text { C. Carey }\end{array}$ & ANU \\
\hline 2009 & Nov. 4-6 & 35 & 35 & 27 & $\begin{array}{l}\text { 1. Business School, UWA } \\
\text { 2. Centre for Economic Policy Research, ANU }\end{array}$ & $\begin{array}{l}\text { K. Clements } \\
\text { Izan }\end{array}$ & S. Pollock & UWA \\
\hline 2010 & Nov. 17-19 & 31 & 31 & 20 & $\begin{array}{l}\text { 1. Research School of Economics, ANU } \\
\text { 2. Business School, UWA } \\
\text { 3. School of Economics, UQ }\end{array}$ & $\begin{array}{l}\text { R. Breunig } \\
\text { R. Cornes } \\
\text { R. Gregory }\end{array}$ & $\begin{array}{l}\text { R. Baillie } \\
\text { B. Lawton }\end{array}$ & ANU \\
\hline
\end{tabular}


TABLE A1 (continued)

DETAILS OF THE CONFERENCES, 1987-2019

\begin{tabular}{|c|c|c|c|c|c|c|c|c|}
\hline Year & Date & $\begin{array}{l}\text { Number } \\
\text { of } \\
\text { students } \\
\text { involved }\end{array}$ & $\begin{array}{l}\text { Number of } \\
\text { discussants } \\
\text { involved }\end{array}$ & $\begin{array}{l}\text { Number of } \\
\text { institutions } \\
\text { involved } \\
\text { (students } \\
\text { and } \\
\text { discussants) }\end{array}$ & Partner institutions & Convenors & Co-ordinators & Location \\
\hline 2011 & Nov. $19-20$ & 36 & 36 & 22 & $\begin{array}{l}\text { 1. School of Economics, UQ } \\
\text { 2. Business School, UWA } \\
\text { 3. Research School of Economics, ANU }\end{array}$ & $\begin{array}{l}\text { G. Lordan } \\
\text { P. Frijters }\end{array}$ & L. West & UQ \\
\hline 2012 & Nov. 7-9 & 30 & 30 & 20 & $\begin{array}{l}\text { 1. Business School, UWA } \\
\text { 2. Research School of Economics, ANU } \\
\text { 3. School of Economics, UQ } \\
\text { 4. Faculty of Business and Economics, Monash }\end{array}$ & $\begin{array}{l}\text { Izan } \\
\text { K. Clements }\end{array}$ & M. Han & UWA \\
\hline 2013 & Nov. 7-9 & 30 & 30 & 20 & $\begin{array}{l}\text { 1. Research School of Economics, ANU } \\
\text { 2. Business School, UWA } \\
\text { 3. School of Economics, UQ } \\
\text { 4. Faculty of Business and Economics, Monash }\end{array}$ & A. Booth & C. Treadwell & ANU \\
\hline 2014 & Nov $12-14$ & 27 & 27 & 18 & $\begin{array}{l}\text { 1. Faculty of Business and Economics, Monash } \\
\text { 2. Business School, UWA } \\
\text { 3. Research School of Economics, ANU } \\
\text { 4. School of Economics, UQ }\end{array}$ & $\begin{array}{l}\text { S. King } \\
\text { P. Maitra } \\
\text { D. Harris }\end{array}$ & N. Calastas & Monash \\
\hline 2015 & Nov $11-13$ & 24 & 25 & 15 & $\begin{array}{l}\text { 1. School of Economics, UQ } \\
\text { 2. Faculty of Business and Economics, Monash } \\
\text { 3. Research School of Economics, ANU } \\
\text { 4. Business School, UWA }\end{array}$ & $\begin{array}{l}\text { P. Frijters } \\
\text { K. Kalayci } \\
\text { H. Yuan }\end{array}$ & S. Brischetto & UQ \\
\hline
\end{tabular}


TABLE A1 (continued)

DETAILS OF THE CONFERENCES, 1987-2019

\begin{tabular}{|c|c|c|c|c|c|c|c|c|}
\hline Year & Date & $\begin{array}{l}\text { Number } \\
\quad \text { of } \\
\text { students } \\
\text { involved }\end{array}$ & $\begin{array}{l}\text { Number of } \\
\text { discussants } \\
\text { involved }\end{array}$ & $\begin{array}{l}\text { Number of } \\
\text { institutions } \\
\text { involved } \\
\text { (students } \\
\quad \text { and } \\
\text { discussants) }\end{array}$ & Partner institutions & Convenors & Co-ordinators & Location \\
\hline 2016 & Nov 9-11 & 32 & 32 & 19 & $\begin{array}{l}\text { 1. Business School, UWA } \\
\text { 2. Research School of Economics, ANU } \\
\text { 3. School of Economics, UQ } \\
\text { 4. Faculty of Business and Economics, Monash }\end{array}$ & $\begin{array}{l}\text { K. Clements } \\
\text { Izan }\end{array}$ & M. Han & UWA \\
\hline 2017 & Nov 1-3 & 34 & 34 & 16 & $\begin{array}{l}\text { 1. University of Melbourne } \\
\text { 2. Business School, UWA } \\
\text { 3. Research School of Economics, ANU } \\
\text { 4. School of Economics, UQ } \\
\text { 5. Faculty of Business and Economics, Monash }\end{array}$ & N. Erkal & & Melb \\
\hline 2018 & Oct 30 - Nov 2 & 32 & 32 & 21 & $\begin{array}{l}\text { 1. Business School, UNSW } \\
\text { 2. University of Melbourne } \\
\text { 3. Business School, UWA } \\
\text { 4. Research School of Economics, ANU } \\
\text { 5. School of Economics, UQ } \\
\text { 6. Faculty of Business and Economics, Monash }\end{array}$ & $\begin{array}{l}\text { G. Foster } \\
\text { S. Lee } \\
\text { W. Tham }\end{array}$ & S. Hickey & UNSW \\
\hline 2019 & Oct 31 - Nov 1 & 32 & 32 & 19 & $\begin{array}{l}\text { 1. Crawford School of Public Policy, ANU } \\
\text { 2. Research School of Economics, ANU } \\
\text { 3. Business School, UWA } \\
\text { 4. Business School, UNSW } \\
\text { 5. School of Economics, UQ } \\
\text { 6. Faculty of Business and Economics, Monash } \\
\text { 7. University of Melbourne }\end{array}$ & $\begin{array}{l}\text { R. Breunig } \\
\text { S. Mangin }\end{array}$ & $\begin{array}{l}\text { L. Ceric } \\
\text { D. Paul }\end{array}$ & ANU \\
\hline Total & & 895 & 892 & 641 & & & & \\
\hline
\end{tabular}


TABLE A2

PRIZES FOR STUDENTS AND DISCUSSANTS, 1995-2019

\begin{tabular}{|c|c|c|c|}
\hline Year & Prize & Winner & Affiliation \\
\hline 1995 & $\begin{array}{l}\text { Best Student Presentation } \\
\text { Best Discussant } \\
\text { Special Prize for Special Contribution as a Discussant }\end{array}$ & $\begin{array}{l}\text { Preston, A. } \\
\text { Martin, V. } \\
\text { Gregory, R. }\end{array}$ & $\begin{array}{l}\text { University of Western Australia } \\
\text { University of Melbourne } \\
\text { Australian National University }\end{array}$ \\
\hline 1996 & $\begin{array}{l}\text { Most Innovative Topic } \\
\text { Special Prize for the First Non-Australian-and-New Zealand Student } \\
\text { Special Recognition for Contributions as a Discussant } \\
\text { Best Discussant } \\
\text { Special Recognition for Contributions as a Discussant }\end{array}$ & $\begin{array}{l}\text { Picton, M. } \\
\text { Muckle, N. } \\
\text { Grant, S. } \\
\text { McLaren, K. } \\
\text { Woodbridge, G. }\end{array}$ & $\begin{array}{l}\text { Monash University } \\
\text { University of Nottingham } \\
\text { Australian National University } \\
\text { Monash University } \\
\text { Australian Competition \& Consumer Comission }\end{array}$ \\
\hline 1997 & $\begin{array}{l}\text { Best Student Presentation } \\
\text { Honourable Mention for Quality Student Presentation } \\
\text { Honourable Mention for Quality Student Presentation } \\
\text { Honourable Mention for Quality Student Presentation } \\
\text { Special Mention for Contribution as a Discussant } \\
\text { Best Discussant } \\
\text { Special Mention for Contribution as a Discussant }\end{array}$ & $\begin{array}{l}\text { Vaithianathan, R. } \\
\text { Swift, R. } \\
\text { Leong, K. } \\
\text { Balatbat, M. } \\
\text { Woodland, A. } \\
\text { Griffiths, B. } \\
\text { Twite, G. }\end{array}$ & $\begin{array}{l}\text { University of Auckland } \\
\text { Griffith University } \\
\text { University of Western Australia } \\
\text { University of Sydney } \\
\text { University of Sydney } \\
\text { University of New England } \\
\text { University of New South Wales }\end{array}$ \\
\hline 1998 & $\begin{array}{l}\text { Best Student Presentation } \\
\text { Best Discussant }\end{array}$ & $\begin{array}{l}\text { Tang, KK. } \\
\text { Doiron, D. }\end{array}$ & $\begin{array}{l}\text { Australian National University } \\
\text { University of Sydney }\end{array}$ \\
\hline 1999 & $\begin{array}{l}\text { Poynton and Partners Business Economics Prize } \\
\text { Honourable Mention } \\
\text { Best Student Presentation } \\
\text { Certificate of Commendation } \\
\text { Certificate of Commendation } \\
\text { Honourable Mention } \\
\text { Best Discussant }\end{array}$ & $\begin{array}{l}\text { Yelten, S. } \\
\text { Chang, H-C. } \\
\text { Dennis, R. } \\
\text { Ma, S. } \\
\text { Salim, V. } \\
\text { Martin, V. } \\
\text { Fiebig, D. }\end{array}$ & $\begin{array}{l}\text { University of Chicago } \\
\text { Australian National University } \\
\text { Australian National University } \\
\text { University of Adelaide } \\
\text { University of Western Australia } \\
\text { University of Melbourne } \\
\text { University of Sydney }\end{array}$ \\
\hline
\end{tabular}


TABLE A2 (continued)

PRIZES FOR STUDENTS AND DISCUSSANTS, 1995-2019

\begin{tabular}{|c|c|c|c|}
\hline Year & Prize & Winner & Affiliation \\
\hline 2000 & $\begin{array}{l}\text { Best Student Presentation } \\
\text { Honourable Mention } \\
\text { Best Discussant } \\
\text { Honourable Mention }\end{array}$ & $\begin{array}{l}\text { Romalis, J. } \\
\text { Melatos, M. } \\
\text { Bewley, R. } \\
\text { Chapman, B. }\end{array}$ & $\begin{array}{l}\text { MIT } \\
\text { University of Sydney } \\
\text { University of New South Wales } \\
\text { Australian National University }\end{array}$ \\
\hline 2001 & $\begin{array}{l}\text { Runner Up Best Presentation } \\
\text { Best Paper in Industrial Economics } \\
\text { Best Paper in Finance } \\
\text { Best Presentation } \\
\text { Runner Up Best Paper in Finance } \\
\text { Runner Up Best Presentation } \\
\text { Runner Up Best Presentation } \\
\text { Runner Up Best Discussant } \\
\text { Best Discussant }\end{array}$ & $\begin{array}{l}\text { Lan, Y. } \\
\text { Dodds, S. } \\
\text { Duncan, P. } \\
\text { Joiner, A. } \\
\text { Pham, P. } \\
\text { Suzuki, T. } \\
\text { Kim, D-K. } \\
\text { Heaney, R. } \\
\text { Anderson, H. }\end{array}$ & $\begin{array}{l}\text { University of Western Australia } \\
\text { Queen's University } \\
\text { University of Newcastle } \\
\text { Monash University } \\
\text { Monash University } \\
\text { Australian National University } \\
\text { University of New South Wales } \\
\text { Australian National University } \\
\text { Monash University }\end{array}$ \\
\hline 2002 & $\begin{array}{l}\text { Runner Up Best Presentation } \\
\text { Best Presentation } \\
\text { Runner Up Best Presentation } \\
\text { Runner Up Best Discussant } \\
\text { Best Discussant }\end{array}$ & $\begin{array}{l}\text { Fogarty, J. } \\
\text { Kam, T. C. Y } \\
\text { Thai, C. } \\
\text { Kompas, T. } \\
\text { Gans, J. }\end{array}$ & $\begin{array}{l}\text { University of Western Australia } \\
\text { University of Melbourne } \\
\text { University of New South Wales } \\
\text { Australian National University } \\
\text { University of Melbourne }\end{array}$ \\
\hline 2003 & $\begin{array}{l}\text { Best Presentation } \\
\text { Best Finance Presentation } \\
\text { Best Finance Presentation } \\
\text { Best Paper in Industrial Economics } \\
\text { Runner Up Best Presentation } \\
\text { Best Paper in Industrial Economics } \\
\text { Best Discussant } \\
\text { Runner Up Best Discussant } \\
\text { Runner Up Best Discussant }\end{array}$ & $\begin{array}{l}\text { Thorp, S. } \\
\text { To, T. D. } \\
\text { Hall, J. } \\
\text { Kohpaiboon, A. } \\
\text { McHugh, Z. } \\
\text { Welch, E. } \\
\text { Bennett, J. } \\
\text { Kingston, G. } \\
\text { Martin, G. }\end{array}$ & $\begin{array}{l}\text { University of New South Wales } \\
\text { University of Technology, Sydney } \\
\text { University of Queensland } \\
\text { Australian National University } \\
\text { Queensland University of Technology } \\
\text { Australian National University } \\
\text { Australian National University } \\
\text { University of New South Wales } \\
\text { Monash University }\end{array}$ \\
\hline
\end{tabular}


TABLE A2 (continued)

PRIZES FOR STUDENTS AND DISCUSSANTS, 1995-2019

\begin{tabular}{|c|c|c|c|}
\hline Year & Prize & Winner & Affiliation \\
\hline 2004 & $\begin{array}{l}\text { Best Presentation } \\
\text { Runner Up Best Presentation } \\
\text { Best Discussant } \\
\text { Runner Up Best Discussant } \\
\text { Runner Up Best Discussant }\end{array}$ & $\begin{array}{l}\text { Wade, K. } \\
\text { Schlichting, D. } \\
\text { Clements, K. } \\
\text { King, S. } \\
\text { Dixon, P. }\end{array}$ & $\begin{array}{l}\text { La Trobe University } \\
\text { University of Sydney } \\
\text { University of Western Australia } \\
\text { Australian Competition \& Consumer Comission } \\
\text { Monash University }\end{array}$ \\
\hline 2005 & $\begin{array}{l}\text { Best Finance Presentation } \\
\text { Best Presentation } \\
\text { Best Economic Presentation } \\
\text { Best Discussant }\end{array}$ & $\begin{array}{l}\text { Forrester, D. } \\
\text { Johnston, D. } \\
\text { Pacheco, G. } \\
\text { Thorp, S. }\end{array}$ & $\begin{array}{l}\text { University of New South Wales } \\
\text { University of Melbourne } \\
\text { University of Auckland } \\
\text { University of Technology Sydney }\end{array}$ \\
\hline 2006 & $\begin{array}{l}\text { Runner Up Best Presentation } \\
\text { Best Presentation } \\
\text { Best Discussant } \\
\text { Runner Up Best Discussant }\end{array}$ & $\begin{array}{l}\text { Headey, D. } \\
\text { Roessler, C. } \\
\text { Leigh, A. } \\
\text { Vahid, F. }\end{array}$ & $\begin{array}{l}\text { University of Queensland } \\
\text { University of Melbourne } \\
\text { Australian National University } \\
\text { Australian National University }\end{array}$ \\
\hline 2007 & $\begin{array}{l}\text { Best Presentation } \\
\text { Best Presentation } \\
\text { Best Discussant }\end{array}$ & $\begin{array}{l}\text { Chen, Y. } \\
\text { Valencia, V. } \\
\text { Dixon, P. }\end{array}$ & $\begin{array}{l}\text { University of Melbourne } \\
\text { University of Melbourne } \\
\text { Monash University }\end{array}$ \\
\hline 2008 & $\begin{array}{l}\text { Best Presentation } \\
\text { Runner Up Best Presentation } \\
\text { Best Discussant }\end{array}$ & $\begin{array}{l}\text { Rohde, N. } \\
\text { Nowak, S. } \\
\text { Clements, K. }\end{array}$ & $\begin{array}{l}\text { University of Queensland } \\
\text { Australian National University } \\
\text { University of Western Australia }\end{array}$ \\
\hline 2009 & $\begin{array}{l}\text { Best Presentation (Economics) } \\
\text { Best Presentation (Economics) } \\
\text { Best Presentation (Economics) } \\
\text { Best Presentation (Finance) } \\
\text { Best Presentation (Finance) - Runner up } \\
\text { Best Discussant } \\
\text { Best Discussant - Runner up }\end{array}$ & $\begin{array}{l}\text { Verani, S. } \\
\text { Gao, G. } \\
\text { Evans, S. } \\
\text { Etheridge, D. } \\
\text { Levy, A. } \\
\text { Foster, D. } \\
\text { Adams, R. }\end{array}$ & $\begin{array}{l}\text { University of California, Santa Barbara } \\
\text { University of Western Australia } \\
\text { Australian National University } \\
\text { University of Western Australia } \\
\text { University of New South Wales } \\
\text { Australian National University } \\
\text { University of Queensland }\end{array}$ \\
\hline
\end{tabular}


TABLE A2 (continued)

PRIZES FOR STUDENTS AND DISCUSSANTS, 1995-2019

\begin{tabular}{|c|c|c|c|}
\hline Year & Prize & Winner & Affiliation \\
\hline 2010 & $\begin{array}{l}\text { Best Student Presentation } \\
\text { Best Student Presentation }\end{array}$ & $\begin{array}{l}\text { de Braganca, G. F. } \\
\text { Ng, J. }\end{array}$ & $\begin{array}{l}\text { Victoria University of Wellington } \\
\text { Monash University }\end{array}$ \\
\hline 2011 & $\begin{array}{l}\text { Best Presentation in Economics } \\
\text { Best Discussant } \\
\text { Best Discussant }\end{array}$ & $\begin{array}{l}\text { Wang, B. } \\
\text { Jensen, P. } \\
\text { Sheen, J. }\end{array}$ & $\begin{array}{l}\text { Macquarie University } \\
\text { University of Melbourne } \\
\text { Macquarie University }\end{array}$ \\
\hline 2012 & $\begin{array}{l}\text { Best Student Presentation (Economics) } \\
\text { Student with Most Potential (Finance) } \\
\text { Best Student Presentation (Finance) } \\
\text { Best Discussant (Economics) } \\
\text { Best Discussant (Economics) } \\
\text { Best Discussant (Finance) } \\
\text { Best Discussant (Finance) }\end{array}$ & $\begin{array}{l}\text { Collins, J. } \\
\text { Liu, Z. } \\
\text { Wang, J. } \\
\text { Cobb-Clark, D. } \\
\text { Strachan, R. } \\
\text { Thorp, S. } \\
\text { Walter, T. }\end{array}$ & $\begin{array}{l}\text { University of Western Australia } \\
\text { University of Queensland } \\
\text { University of Sydney } \\
\text { University of Melbourne } \\
\text { Australian National University } \\
\text { University of Technology Sydney } \\
\text { SIRCA }\end{array}$ \\
\hline 2013 & $\begin{array}{l}\text { Best Student Presentation } \\
\text { Best Discussant }\end{array}$ & $\begin{array}{l}\text { Hall, J. } \\
\text { Ray, R. }\end{array}$ & $\begin{array}{l}\text { University of New South Wales } \\
\text { Monash University }\end{array}$ \\
\hline 2014 & $\begin{array}{l}\text { Best Student Presentation } \\
\text { Best Discussant } \\
\text { Best Discussant }\end{array}$ & $\begin{array}{l}\text { Burfurd, I. } \\
\text { Williams, J. } \\
\text { Clements, K. }\end{array}$ & $\begin{array}{l}\text { University of Melbourne } \\
\text { University of Melbourne } \\
\text { University of Western Australia }\end{array}$ \\
\hline 2015 & $\begin{array}{l}\text { Best Presentation } \\
\text { Best Discussant }\end{array}$ & $\begin{array}{l}\text { Gauriot, R. } \\
\text { Foster, G. }\end{array}$ & $\begin{array}{l}\text { Queensland University of Technology } \\
\text { University of New South Wales }\end{array}$ \\
\hline 2016 & $\begin{array}{l}\text { Best Presentation, Economics } \\
\text { Best Presentation, Finance } \\
\text { Best Discussant, Economics } \\
\text { Best Discussant, Finance } \\
\text { Best Discussant, Finance }\end{array}$ & $\begin{array}{l}\text { Delacrétaz, D. } \\
\text { Zhang, E. } \\
\text { Frijters, P. } \\
\text { Walter, T. } \\
\text { Dungey, M. }\end{array}$ & $\begin{array}{l}\text { University of Melbourne } \\
\text { University of New South Wales } \\
\text { University of Queensland } \\
\text { University of Sydney } \\
\text { University of Tasmania }\end{array}$ \\
\hline
\end{tabular}


TABLE A2 (continued)

PRIZES FOR STUDENTS AND DISCUSSANTS, 1995-2019

\begin{tabular}{|c|c|c|c|}
\hline Year & Prize & Winner & Affiliation \\
\hline 2017 & $\begin{array}{l}\text { Best Paper in Finance } \\
\text { Best Paper in Economics } \\
\text { Best Discussant }\end{array}$ & $\begin{array}{l}\text { Drummond, P. } \\
\text { Koh, B. } \\
\text { Foster, G. }\end{array}$ & $\begin{array}{l}\text { Australian National University } \\
\text { University of Melbourne } \\
\text { University of New South Wales }\end{array}$ \\
\hline 2018 & $\begin{array}{l}\text { Best Presentation } \\
\text { Best Discussant }\end{array}$ & $\begin{array}{l}\text { Deutscher, N. } \\
\text { Jetter, M. }\end{array}$ & $\begin{array}{l}\text { Australian National University } \\
\text { University of Western Australia }\end{array}$ \\
\hline 2019 & $\begin{array}{l}\text { Best Paper } \\
\text { Best Discussant }\end{array}$ & $\begin{array}{l}\text { Griselda, S. } \\
\text { Johnston, D. }\end{array}$ & $\begin{array}{l}\text { University of Melbourne } \\
\text { Monash University }\end{array}$ \\
\hline
\end{tabular}


TABLE A3

SPECIAL LECTURES AND AFTER-DINNER SPEECHES, 1987-2019

\begin{tabular}{|c|c|c|c|}
\hline Year & Name & Institution & Topic \\
\hline 1987 & $\begin{array}{l}\text { Hyde, J. } \\
\text { Jonson, P. }\end{array}$ & $\begin{array}{l}\text { IPA } \\
\text { Reserve Bank of Australia }\end{array}$ & $\begin{array}{l}\text { Democracy and the Market for Ideas } \\
\text { Professional Economists in Business and Government }\end{array}$ \\
\hline 1988 & $\begin{array}{l}\text { Giles, D. } \\
\text { Gregory, R. } \\
\text { Morgan, D. }\end{array}$ & $\begin{array}{l}\text { University of Canterbury } \\
\text { Australian National University } \\
\text { Commonwealth Treasury }\end{array}$ & $\begin{array}{l}\text { Recent Developments in Econometrics: Lessons for Applied Economists } \\
\text { Jobs and Gender: A Lego Approach to the Labour Market } \\
\text { Economists in Government }\end{array}$ \\
\hline 1989 & $\begin{array}{l}\text { Powell, A. } \\
\text { Shann, E. }\end{array}$ & $\begin{array}{l}\text { University of Melbourne } \\
\text { Access Economics }\end{array}$ & $\begin{array}{l}\text { The Reconciliation of a Computable General Equilibrium and Macroeconomic Modelling: Grounds for Hope? } \\
\text { Economics and Religion }\end{array}$ \\
\hline 1991 & $\begin{array}{l}\text { Blundell-Wignall, A. } \\
\text { Gregory, R. }\end{array}$ & $\begin{array}{l}\text { Reserve Bank of Australia } \\
\text { Australian National University }\end{array}$ & $\begin{array}{l}\text { The Relevance of Modern Macroeconomics to Policy Making in OECD Countries } \\
\text { Job Creation in the Male and Female Workforces in Australia }\end{array}$ \\
\hline 1992 & $\begin{array}{l}\text { Cole, } \mathrm{T} . \\
\text { Creedy, J. }\end{array}$ & $\begin{array}{l}\text { Australian Treasury } \\
\text { University of Melbourne }\end{array}$ & $\begin{array}{l}\text { Challenges as an Economist in the Public Sector } \\
\text { Income Distribution within a General Equilibrium Framework }\end{array}$ \\
\hline 1993 & $\begin{array}{l}\text { Hughes, H. } \\
\text { Mundell, R. } \\
\text { Stoekel, A. }\end{array}$ & $\begin{array}{l}\text { Australian National University } \\
\text { Columbia University } \\
\text { Centre for International Economics }\end{array}$ & $\begin{array}{l}\text { The Lessons of Development } \\
\text { Regionalism vs Multilateralism in the World Economy } \\
\text { The Experience with Deregulation in Mexico and New Zealand and its Implications for Australia }\end{array}$ \\
\hline 1994 & McKibbin, W. & Australian National University & Carbon Taxes and the World Economy \\
\hline 1995 & $\begin{array}{l}\text { Hilmer, F. } \\
\text { Powell, A. }\end{array}$ & $\begin{array}{l}\text { University of New South Wales } \\
\text { Monash University }\end{array}$ & $\begin{array}{l}\text { Competitive Policy: Underlying Ideas and Issues } \\
\text { Terminal Omphaloskepsis? Or, Are Theorists Getting too Much of the Action in Economics? }\end{array}$ \\
\hline
\end{tabular}


TABLE A3 (continued)

SPECIAL LECTURES AND AFTER-DINNER SPEECHES, 1987-2019

\begin{tabular}{|c|c|c|c|}
\hline Year & Name & Institution & Topic \\
\hline 1996 & $\begin{array}{l}\text { Chapman, B. } \\
\text { Harper, I. }\end{array}$ & $\begin{array}{l}\text { Australian National University } \\
\text { University of Melbourne }\end{array}$ & $\begin{array}{l}\text { Economics Research and Public Policy Making: HECS as a Case Study } \\
\text { Directions and Trade-offs - the Future of Australian Academic Life }\end{array}$ \\
\hline 1997 & $\begin{array}{l}\text { Dixon, P. } \\
\text { Smith, T. }\end{array}$ & $\begin{array}{l}\text { Monash University } \\
\text { University of New South Wales }\end{array}$ & $\begin{array}{l}\text { Where Do Old TVs Go? } \\
\text { The Current State and Future Directions of Research in Finance }\end{array}$ \\
\hline 1998 & Gruen, D. & Reserve Bank of Australia & Why does the Australian Dollar Move so Closely with the Terms of Trade? \\
\hline 1999 & $\begin{array}{l}\text { Bewley, R. } \\
\text { King, S. }\end{array}$ & $\begin{array}{l}\text { University of New South Wales } \\
\text { University of Melbourne }\end{array}$ & The Economics of Lobbying (and the Lobbying of Economics) \\
\hline 2000 & Maddock, R. & Business Council of Australia & Cashing in on your Economics \\
\hline 2001 & $\begin{array}{l}\text { Clements, K. } \\
\text { Cameron, L. }\end{array}$ & $\begin{array}{l}\text { University of Western Australia } \\
\text { University of Melbourne }\end{array}$ & $\begin{array}{l}\text { Challenges of Fundraising for a Conference } \\
\text { Staying Sane While Doing Economics: Enlivening the Dismal Science }\end{array}$ \\
\hline 2002 & Watt, I. & Dept. of Finance and Admin & Commonwealth Government Budgets \\
\hline 2003 & Tourky, R. & University of Melbourne & Party Formation in Collective Decision Making \\
\hline 2004 & $\begin{array}{l}\text { Oster, A. } \\
\text { Clements, K. }\end{array}$ & $\begin{array}{l}\text { National Australia Bank } \\
\text { University of Western Australia }\end{array}$ & $\begin{array}{l}\text { The Art and Science of Forecasting the Australian Economy } \\
\text { Tribute to Bob Gregory and his involvement in the PhD Conference }\end{array}$ \\
\hline 2005 & $\begin{array}{l}\text { Barnaba, } \mathrm{M} . \\
\text { Clements, } \mathrm{K} \text {. }\end{array}$ & $\begin{array}{l}\text { University of Western Australia } \\
\text { University of Western Australia }\end{array}$ & $\begin{array}{l}\text { Interplay between Academia and Industry, and the Value of a PhD in Business or Economics } \\
\text { Economic Aspects of Marijuana }\end{array}$ \\
\hline 2006 & Harper, I. & Fair Pay Commission & A Career as an Economist \\
\hline
\end{tabular}


TABLE A3 (continued)

SPECIAL LECTURES AND AFTER-DINNER SPEECHES, 1987-2019

\begin{tabular}{|c|c|c|c|}
\hline Year & Name & Institution & Topic \\
\hline 2007 & $\begin{array}{l}\text { Brennan, G. } \\
\text { Langoulant, J. }\end{array}$ & $\begin{array}{l}\text { Australian National University } \\
\text { WA Chamber of Commerce and Industry }\end{array}$ & $\begin{array}{l}\text { Discounting the Future } \\
\text { Bread and Circuses }\end{array}$ \\
\hline 2008 & McKibbin, W. & Australian National University & Design of Emission Trading Systems \\
\hline 2009 & Frijters, P. & Queensland University of Technology & The Cult of Theoi: Economic Uncertainty and Sacrifices \\
\hline 2010 & Gai, P. & Australian National University & Achieving Financial Stability in a Post GFC World: Lessons from the Coalface \\
\hline 2011 & $\begin{array}{l}\text { Gregory, R. } \\
\text { Edwards, J. }\end{array}$ & $\begin{array}{l}\text { Australian National University } \\
\text { Reserve Bank of Australia }\end{array}$ & \\
\hline 2012 & - & & \\
\hline 2013 & van Ours, J. & University of Melbourne & Marijuana and Economic Behaviour \\
\hline 2014 & Simon, J. & Reserve Bank of Australia & So you've earned a $\mathrm{PhD}$ - now what? \\
\hline 2015 & Clements, $\mathrm{K}$. & University of Western Australia & The Economics Industry \\
\hline 2016 & - & & \\
\hline 2017 & Holden, R. & University of New South Wales & Markets with Network Externalities \\
\hline 2018 & Harper, I. & University of Melbourne & Reflections of a Meddlesome Economist \\
\hline 2019 & Charlton, A. & AlphaBeta Advisors Pty Ltd & What Makes a Good Career? \\
\hline
\end{tabular}




\begin{tabular}{|c|c|c|c|c|c|c|c|c|}
\hline & Name & Affiliation & & Name & Affiliation & & Name & Affiliation \\
\hline & $\underline{1987}$ & & 45. & McCormack, D. & LTb & 90. & Tian, G. & MacQ \\
\hline 1. & Abayasiri-Silva, K. & Monash & 46. & Menon, J. & Melb & 91. & Wan, A. & C'bury \\
\hline 2. & Adams, P. & Melb & 47. & Rahaman, R. & ANU & 92. & Webber, A. & UNSW \\
\hline 3. & Bakalis, S. & LTb & 48. & Rimmer, M. & Melb & 93. & Wild, P. & UQ \\
\hline 4. & Bandara, J. & LTb & 49. & Stonecash, R. & UNSW & 94. & Yang, M. & UNSW \\
\hline 5. & Coxhead, I. & ANU & 50. & Twite, G. & UNSW & 95. & Zhang, X. & Adel \\
\hline 6. & Davidson, B. & UNE & 51. & Wan, G. & UNE & & & \\
\hline 7. & Delforce, J. & UNE & 52. & Wu, P. & Monash & & $\underline{1993}$ & \\
\hline 8. & Gordon, J. & MacQ & 53. & Wu, Y. & Adel & 96. & Ablett, J. & UNSW \\
\hline 9. & Gross. E. & UNSW & 54. & Zhao, B. & UNSW & 97. & Alminaza-Varua, E. & UNE \\
\hline 10. & Gruen, D. & ANU & & & & 98. & Bhar, R. & UWS \\
\hline 11. & Hossaub, A. & $\mathrm{LTb}$ & & $\underline{1991}$ & & 99. & Booth, R. & Monash \\
\hline 12. & Jafforullah, M. & Adel & 55. & Austria, $\overline{M .}$ & ANU & 100. & Buetre, B. & USyd \\
\hline 13. & Kurnuma, Y. & N'cast & 56. & Brooks, R. & Monash & 101. & Campbell, N. & ANU \\
\hline 14. & Liesch, P. & UQ & 57. & Chaiyindeepum, S. & ANU & 102. & Chang, C.-A. & UWA \\
\hline 15. & Manzur, M. & UWA & 58. & Chen, D.L. & UWA & 103. & Daly, K. & UWS \\
\hline 16. & Mumford, K. & MacQ & 59. & da Silva Rosa, R. & UWA & 104. & Ganguli, P. & USyd \\
\hline 17. & O'Donnell, C. & USyd & 60. & Dehnert, J. & UNSW & 105. & Нао, K. & Monash \\
\hline 18. & O'Sullivan, P. & UNSW & 61. & Dwyer, J. & Grif & 106. & Hewitson, G. & $\mathrm{LTb}$ \\
\hline 19. & Panoutsopoulos, V. & ANU & 62. & Grimes, P. & ANU & 107. & Hunter, B. & ANU \\
\hline 20. & Peng, Z.-Y. & Adel & 63. & Gschwind, D. & UQ & 108. & Jubb, C. & Melb \\
\hline 21. & Sharma, K. & UNE & 64. & Han, S. & USyd & 109. & Lim, S. & Adel \\
\hline 22. & Suphachalasai, S. & ANU & 65. & Hawke, A. & ANU & 110. & McBride, P. & Melb \\
\hline & & & 66. & Hopkins, S. & UTas & 111. & Morley, C. & RMIT \\
\hline & $\underline{1988}$ & & 67. & Kim, C D. & ANU & 112. & Naqvi, F. & Monash \\
\hline 23. & Chia, T.-T. & ANU & 68. & Lee, J. & Monash & 113. & Neal, P. & Adel \\
\hline 24. & Dwyer, J. & UQ & 69. & Malakellis, M. & Monash & 114. & Paice, C. & ANU \\
\hline 25. & Firdausy, C. & N'cast & 70. & Mangiri, K. & Grif & 115. & Stewart, M. & Melb \\
\hline 26. & Gruen, D. & ANU & 71. & Meng, X. & ANU & 116. & Suwandi, T. & ANU \\
\hline 27. & Jones, C. & ANU & 72. & Petchey, J. & ANU & 117. & Teesama, G. & UNE \\
\hline 28. & Kaine-Jones, G. & UNE & 73. & Shan, Z. & MacQ & 118. & Teixeira, A. & Auck \\
\hline 29. & Lye, J. & C'bury & 74. & Shi, H.-L. & Monash & 119. & Walsh, D. & Curtin \\
\hline 30. & Moshirian, F. & Monash & 75. & Small, J. & C'bury & 120. & Woo, L.-A. & UNSW \\
\hline 31. & Nakatika, T. & Hito & & & & & & \\
\hline 32. & Salerian, J. & UWA & & $\underline{1992}$ & & & 1994 & \\
\hline 33. & Silby, $\mathrm{H}$. & Melb & 76. & de Jager, G. & UTS & 121. & Aswicahyono, $\mathrm{H}$. & ANU \\
\hline 34. & Suh, C. & UNSW & 77. & Fleming, G. & Auck & 122. & Bateman, $\mathrm{H}$. & UNSW \\
\hline 35. & Tan, $\mathrm{K}$. & USyd & 78. & Fung, T. & ANU & 123. & Bradbury, B. & UNSW \\
\hline 36. & Vanzetti, D. & $\mathrm{LTb}$ & 79. & Huang, Y. & ANU & 124. & Bull, A. & Grif \\
\hline 37. & Yang, Y.Z. & ANU & 80. & Laplagne, P. & UNE & 125. & Chand, S. & ANU \\
\hline & & & 81. & Moore, G. & $\mathrm{LTb}$ & 126. & Diessel, S. & N'cast \\
\hline & $\underline{1989}$ & & 82. & Nahm, D. & USyd & 127. & Divisekera, S. & $\mathrm{LTb}$ \\
\hline 38. & Abraham, D. & ANU & 83. & Nandan, G. & ANU & 128. & Dowling, B. & Melb \\
\hline 39. & Bhatti, I. & Monash & 84. & Onchoke, S. & UNE & 129. & Kim, J. & USyd \\
\hline 40. & Bryant, T. & USyd & 85. & Peter, M. & Monash & 130. & Kim, S.-J. & USyd \\
\hline 41. & Daly, A. & ANU & 86. & Robertson, K. & UWA & 131. & Krasachat, W. & UNE \\
\hline 42. & Hendrix, $\mathrm{H}$. & UQ & 87. & Rose, C. & USyd & 132. & Lee, J. H. & W'gong \\
\hline 43. & Johnson, J. & UWA & 88. & Rummery, S. & ANU & 133. & Lock, C. & UTas \\
\hline 44. & Kawaguchi, A. & ANU & 89. & Smith, H. & ANU & 134. & Locke, C. & USyd \\
\hline
\end{tabular}


TABLE A4 (continued)

THE STUDENTS, 1987-2019

\begin{tabular}{|c|c|c|c|c|c|c|c|c|}
\hline & Name & Affiliation & & Name & Affiliation & & Name & Affiliation \\
\hline 135. & Lumley, S. & $\mathrm{LTb}$ & 180 & Delpachitra, S. & USQ & 227. & Ye, Q. & UWA \\
\hline 136. & Maclean, G. & Auck & 181. & Hewarathna, R. & LTb & & & \\
\hline 137. & Murray, J. & USyd & 182. & Hossain, M. & ANU & & $\underline{1998}$ & \\
\hline 138. & Ng, S. & Monash & 183. & McNeill, J. & UNE & 228. & Centeno, L. & UNE \\
\hline 139. & Robinson, D. & USQ & 184 & Meagher, K. & ANU & 229. & de Francesco, A. & UNSW \\
\hline 140. & Rodriguez, U. & ANU & 185 & Muckle, N. & Nott'ham & 230. & Edge, R. & Princeton \\
\hline 141. & Sarpturk, N. & Deakin & 186. & Nana, G. & VU.W'ton & 231. & Gopal Das, G. & Monash \\
\hline 142. & Tisato, P. & Adel & 187. & Picton, M. & Monash & 232. & Govindarajalu, S. & Curtin \\
\hline 143. & Tourky, R. & UQ & 188 & Purcal, S. & UNSW & 233. & Groen, L. & UTS \\
\hline 144. & Wilson, L. & $\mathrm{LTb}$ & 189 & Robson, A. & UQ & 234. & Heath, A. & LSE \\
\hline & & & 190 & Sequeira, J. & UWA & 235. & Lam, R. & Yale \\
\hline & $\underline{1995}$ & & 191. & Sharma, K. & CSU & 236. & Levtchenkova, S. & ANU \\
\hline 145. & Abello, L.A. & ANU & 192 & Siksamat, S. & Monash & 237. & Martin, Y.-M. & ANU \\
\hline 146. & Beeson, M. & Murdoch & 193. & Vicary, A. & MacQ & 238. & Matthews, K. & MacQ \\
\hline 147. & Bhattacharya, M. & UTas & 194 & Vujanovic, P. & UNSW & 239. & Messings, G. & Melb \\
\hline 148. & Bird, K. & ANU & 195 & Will, L. & ANU & 240. & Monsingh, V. & Curtin \\
\hline 149. & Butler, D. & UWA & & & & 241. & Oktaviani, R. & USyd \\
\hline 150. & Cahill, P. & Murdoch & & $\underline{1997}$ & & 242. & Reiman, C. & C'berra \\
\hline 151. & Cumberworth, M. & UNSW & 196. & Adkins, $\overline{G .}$ & USQ & 243. & Spring, D. & $\mathrm{LTb}$ \\
\hline 152. & Dayanandan, A. & USyd & 197. & Ahmed, N. & USyd & 244. & Ssewanyana, S. & USyd \\
\hline 153. & Dungey, M. & ANU & 198 & Arm Liu, Yuk Chu & ANU & 245. & Strachan, R. & Monash \\
\hline 154. & Fry, S. & UTas & 199 & Balatbat, M. & USyd & 246. & Tang, K. & ANU \\
\hline 155. & Hutson, E. & UTS & 200 & Blacklow, P. & UTas & 247. & Trigger, D. & C'berra \\
\hline 156. & Kalb, G. & Monash & 201. & Boymal, J. & Monash & 248. & Tseng, Y.-P. & ANU \\
\hline 157. & Laskar, M. & Monash & 202. & Clarke, I. & N'cast & 249. & Wittwer, G. & Adel \\
\hline 158. & Marsden, A. & Auck & 203. & Dancer, D. & USyd & 250. & Yang, W. & UWA \\
\hline 159. & Martin, G. & Monash & 204 & Durbarry, R. & Nott'ham & 251. & Zhao, X. & UNE \\
\hline 160. & McDonald, J. & Melb & 205. & Gallery, G. & UQ & & & \\
\hline 161. & Nishat, M. & Auck & 206. & Ginting, E. & Monash & & 1999 & \\
\hline 162. & Ong, L.L. & UWA & 207. & Gray, M. & ANU & 252. & Ackland, N. & USyd \\
\hline 163. & Preston, A. & UWA & 208. & Handley, J. & Melb & 253. & Ackland, R. & ANU \\
\hline 164. & Ralston, D. & Bond & 209. & Lee, C.S. & ANU & 254. & Akmal, M. & ANU \\
\hline 165. & Roca, E. & Grif & 210. & Lee, Y.L. & UWA & 255. & Auepiyachut, W. & W'gong \\
\hline 166. & Rogers, M. & ANU & 211. & Leong, K. & UWA & 256. & Becker, R. & QUT \\
\hline 167. & Schmidt, G. & Monash & 212 & Mahadevan, R. & ANU & 257. & Blackwell, B. & UQ \\
\hline 168. & Strutt, A. & Adel & 213. & Morrison, D. & UQ & 258. & Caspersz, D. & UWA \\
\hline 169. & Sweeney, M. & Melb & 214. & Njuguna, A. & UNE & 259. & Chang, H.-C. & ANU \\
\hline 170. & Tan, Clarence & Bond & 215 & Olive, M. & Curtin & 260. & Crompton, P. & UWA \\
\hline 171. & Valenzuela, M.R. & UNE & 216 & Oppenheim, P. & Monash & 261. & Dennis, R. & ANU \\
\hline 172. & Wen, M. & Monash & 217 & Pattenden, K. & UNSW & 262. & Digal, L. & USyd \\
\hline & & & 218 & Pinder, S. & Monash & 263. & Elerian, O. & Oxford \\
\hline & $\underline{1996}$ & & 219 & Qiu, M. & UWA & 264. & Foster, N. & Nott'ham \\
\hline 173. & Atukorala, R. & Monash & 220 & Ragunathan, V. & RMIT & 265. & Guest, O. & $\mathrm{LTb}$ \\
\hline 174. & Barkatullah, N. & USyd & 221. & Salayo, N. & Grif & 266. & Hart, A. & USyd \\
\hline 175. & Beg, R. & $\mathrm{LTb}$ & 222 & Suryahadi, A. & ANU & 267. & He, X.-Z. & UTS \\
\hline 176. & Cardak, B. & Melb & 223 & Swift, R. & Grif & 268. & Ho, V.-T. & Monash \\
\hline 177. & Chen, $\mathrm{T}$. & ANU & 224 & Tan, Christine & Melb & 269. & Johnson, P. & UWA \\
\hline 178. & Cheung, L. & W'gong & 225 & Vaithianathan, R. & Auck & 270. & Kedir, A. & Nott'ham \\
\hline 179. & Davidson, S. & RMIT & 226 & van de Vyver, M. & UWA & 271. & Kong, $\mathrm{X}$. & USyd \\
\hline
\end{tabular}


TABLE A4 (continued)

THE STUDENTS, 1987-2019

\begin{tabular}{|c|c|c|c|c|c|c|c|c|}
\hline \multicolumn{2}{|r|}{ Name } & \multirow{2}{*}{$\begin{array}{l}\text { Affiliation } \\
\text { UNE }\end{array}$} & \multicolumn{2}{|r|}{ Name } & \multirow{3}{*}{$\begin{array}{l}\text { Affiliation } \\
\text { VU.W'ton } \\
\text { UQ }\end{array}$} & \multicolumn{2}{|r|}{ Name } & \multirow{2}{*}{ Affiliation } \\
\hline 272. & Kortt, M. & & 317. & Claus, I. & & 364. & Kam, T. & \\
\hline 273. & Le, P. & Grif & 318 & Collins, D. & & 365. & Kao, T. & Auck \\
\hline 274. & Ma, S. & Adel & 319 & Costello, G. & UWA & 366. & Kim, J. J. M. & ANU \\
\hline 275. & Pavlov, V. & ANU & 320 & Cottet, R. & USyd & 367. & Mathysen, H. & Monash \\
\hline 276. & Salim, V. & UWA & 321. & Dodds, S. & Queen's & 368. & McCulloch, J. & UTS \\
\hline 277. & Savage, S. & Curtin & 322 & Duncan, P. & N'cast & 369. & Merhebi, R. & UNSW \\
\hline 278. & Shiu, A. & UNSW & 323 & Fry, R. & Melb & 370. & Pershin, V. & UWA \\
\hline 279. & Tupou, W. & Deakin & 324 & Hisarciklilar, M. & Nott'ham & 371. & Pilcher, R. & CSU \\
\hline 280. & Weier, A. & Monash & 325 & Howell, B. & VU.W'ton & 372. & Reedman, L. & N'cast \\
\hline 281. & Wilkins, R. & Melb & 326 . & Jensen, P. & UNSW & 373. & Saleh, A. & W'gong \\
\hline 282. & Yelten, S. & Chicago & 327 & Joiner, A. & Monash & 374. & Scutella, R. & Melb \\
\hline 283. & Zhang, Q. & UTas & 328 & Kem Reat, V. & ANU & 375. & Spong, $\mathrm{H}$. & RMIT \\
\hline & & & 329 & Kim, D.-K. & UNSW & 376. & Sreedharan, N. & Curtin \\
\hline & $\underline{2000}$ & & 330 & Lan, Y. & UWA & 377. & Straton, A. & UQ \\
\hline 284. & Arumanayagam, S. & Monash & 331. & Lee, P. & USyd & 378. & Sun, C. H. & ANU \\
\hline 285. & Asano, A. & ANU & 332 & Lenten, L. & $\mathrm{LTb}$ & 379. & Thai, C. & UNSW \\
\hline 286. & Bandara, Y. & UQ & 333. & Loundes, J. & Melb & & & \\
\hline 287. & Dobbie, M. & MacQ & 334 & Meyer-Boehm, G. & Grif & & $\underline{2003}$ & \\
\hline 288. & Dockery, M. & Curtin & 335 & O'Shannassy, T. & Monash & 380. & Almeida, $\mathrm{N}$. & UNSW \\
\hline 289. & Hendrie, D. & UWA & 336. & Pham, P. & Monash & 381. & Carvalho, J. P. & UWA \\
\hline 290. & Jiang, $\mathrm{T}$. & ANU & 337. & Richardson, C. & UTas & 382. & Chandra, M. & UWA \\
\hline 291. & Kaluge, D. & C'berra & 338 & Seelajaroen, R. & ANU & 383. & Concu,G. & UWA \\
\hline 292. & Kwon, O.-K. & UTS & 339 & Shacklock, K. & Grif & 384. & Foley, D. & UQ \\
\hline 293. & Lawrance, $\mathrm{T}$. & UNSW & 340 & Skamnelos, I. & Nott'ham & 385. & Gunn, L. & Monash \\
\hline 294. & Lee, H.-L. & Monash & 341. & Sutummakid, N. & MacQ & 386. & Gupta, A. & Adel \\
\hline 295. & Melatos, M. & USyd & 342 & Suzuki, T. & ANU & 387. & Hajargasht, G. & UQ \\
\hline 296. & Menzies, G. & Oxford & 343 & Taylor, S. & UTS & 388. & Hall, J. & UQ \\
\hline 297. & Nguyen, D. & W'gong & 344 & van der Merwe, R. & Curtin & 389. & Jaleel, F. & MacQ \\
\hline 298. & O'Brien, M. & N'cast & 345 & Voola, J. & Curtin & 390. & Kohpaiboon, A. & ANU \\
\hline 299. & Perry, N. & $\mathrm{LTb}$ & 346. & Wilson, J. & Adel & 391. & Le, H. G. & ANU \\
\hline 300. & Romalis, J. & MIT & 347 & Winston, A. & Monash & 392. & Leu, C. Y. & USyd \\
\hline 301. & Shami, R. & Monash & 348. & Wood, B. & UWA & 393. & Lise, J. & Queen's \\
\hline 302. & Sidorenko, A. & ANU & & & & 394. & Maheswaran, K. & Melb \\
\hline 303. & Soucik, V. & ECU & & $\underline{2002}$ & & 395. & Mangano, M. & Curtin \\
\hline 304. & Stachurski, J. & Melb & 349 & Cavoli, $\overline{\mathrm{T} .}$ & Adel & 396. & McHugh, Z. & QUT \\
\hline 305. & Temur, M. & UNSW & 350 & Chan, F. & UWA & 397. & Naranpanawa, A. & Grif \\
\hline 306. & Viney, R. & USyd & 351. & Chen, Z. & UNSW & 398. & Nguyen, O. & Grif \\
\hline 307. & $\mathrm{Vu}, \mathrm{Q}$. & ANU & 352 & Chong, H. H. & Curtin & 399. & Parasnis, J. & Monash \\
\hline 308. & Wait, A. & ANU & 353 & Clarke, A. & McMaster & 400. & Shareef, R. & UWA \\
\hline & & & 354. & Culas, R. J. & USyd & 401. & Siow, A. & UNSW \\
\hline & $\underline{2001}$ & & 355. & Dark, J. & N'cast & 402. & Smiles, S. & ANU \\
\hline 309. & Abrantes, R. & Chicago & 356. & Eckerman, S. & UNSW & 403. & Smith, B. & UWA \\
\hline 310. & Agalewatte, T. & W'gong & 357. & Ellis, L. & UNSW & 404. & Smith, P. & Melb \\
\hline 311. & Ahn, K. & S.K.Kwan & 358 & Eto, $\mathrm{T}$. & ANU & 405. & Taylor, N. & Murdoch \\
\hline 312. & Bugeja, M. & USyd & 359 & Fogarty, J. & UWA & 406. & Thorp, S. & UNSW \\
\hline 313. & Cai, L. & ANU & 360 & Funston, K. & ANU & 407. & To, T. D. & UTS \\
\hline 314. & Cheung, S. & USyd & 361. & Gang, L. & UTas & 408. & Tooth, R. & UNSW \\
\hline 315. & Chng, M. & Melb & 362 & Ghosh, P. C. & USyd & 409. & Venn, D. & Melb \\
\hline 316. & Chu, H. & UTS & 363. & Iyer, L. & MIT & 410. & Villano, R. & UNE \\
\hline
\end{tabular}


TABLE A4 (continued)

THE STUDENTS, 1987-2019

\begin{tabular}{|c|c|c|c|c|c|c|c|c|}
\hline \multicolumn{2}{|r|}{ Name } & \multirow{3}{*}{$\begin{array}{l}\text { Affiliation } \\
\text { ANU }\end{array}$} & \multicolumn{2}{|r|}{ Name } & Affiliation & \multicolumn{2}{|r|}{ Name } & Affiliation \\
\hline 411. & Welch, E. & & 456 & Pontines, V. & Adel & 501. & Gould, J. & UWA \\
\hline & & & 457 & Ramful, P. & Monash & 502 & Haq, M. & RMIT \\
\hline & $\underline{2004}$ & & 458 & Shan, Y. & UNSW & 503 & Hoque, M.A. & Curtin \\
\hline 412. & Ahmed, A.D. & Melb & 459 & Strickland, C. & Monash & 504 & Jarman, B. & USyd \\
\hline 413. & Carroll, N. & ANU & 460 & Tang, S. & Grif & 505 & Lee, A. & UNSW \\
\hline 414. & Greenville, J. & USyd & 461 & Walker, D. & $\mathrm{LTb}$ & 506 & MacLaine, D. & UQ \\
\hline 415. & Hayes, R. & Melb & 462 & Wheeler, S. & USA & 507 & Manning, M. & USyd \\
\hline 416. & Hayman, P. & USyd & 463 & Williams, A. & UWA & 508 & Naiker, V. & Auck \\
\hline 417. & Hung, P.V. & USyd & 464 & Woo, W.C. & Monash & 509 & O'Brien, M. & UQ \\
\hline 418. & Iyer, K. & UQ & 465 & Yahyaee, K.A. & UNSW & 510 & Oum, S. & Monash \\
\hline 419. & Jongwanich, J. & ANU & 466 & Zhu, Z.H. & VU.W'ton & 511. & Powell, R. & ECU \\
\hline 420. & Khan, H. & Melb & & & & 512 & Ryder, B. & UQ \\
\hline 421. & Kilhaule, A. & Curtin & & $\underline{2006}$ & & 513 & Shauki, A. & USA \\
\hline 422. & Kim, G. & Monash & 467 & Angus, $\overline{S .}$ & UNSW & 514 & Siminski, P. & UNSW \\
\hline 423. & Kutschukian, J.M. & USyd & 468 & Anukoonwattaka, W. & Melb & 515 & Smith, G. & UWA \\
\hline 424. & Leow, I. & UNSW & 469 & Bhattacharyya, S. & ANU & 516 & Sun, S. & ANU \\
\hline 425. & Libich, J. & UNSW & 470 & Broderick, S. & UNE & 517 & Tharavanij, P. & Monash \\
\hline 426. & Lynch, T. & $\mathrm{LTb}$ & 471 & Bugden, J. & $\mathrm{LTb}$ & 518 & Valencia, V. & Melb \\
\hline 427. & Milunovich, G. & UNSW & 472 & Buncic, D. & UNSW & 519 & Vu, H. & Melb \\
\hline 428. & Neal, M. & USyd & 473 & Byford, M. & Melb & 520 & Wei, Z. & Adel \\
\hline 429. & Pambudi, D. & Monash & 474 & Cornwell, K. & Monash & 521. & Yan, Y.H. & UTas \\
\hline 430. & Peng, $\mathrm{X}$. & Monash & 475 & Dang, L. & Melb & 522 & Zhang, H.F. & Melb \\
\hline 431. & Schlichting, D. & USyd & 476 & Davis, $\mathrm{T}$. & USyd & 523. & Zhang, X. & Monash \\
\hline 432. & Sinha, K. & ANU & 477 & Djumashev, R. & Monash & & & \\
\hline 433. & Sirasoontorn, P. & ANU & 478. & Gebermedhin, T.A. & USyd & & $\underline{2008}$ & \\
\hline 434. & Verikios, G. & UWA & 479 . & Greenaway-McGrevy, R. & Auck & 524. & Ainsworth, A. & UNSW \\
\hline 435. & Wade, $\mathrm{K}$. & $\mathrm{LTb}$ & 480. & Headey, D. & UQ & 525 & Chatterjee, I. & Monash \\
\hline 436. & White, S. & QUT & 481. & Koh, S. & UWA & 526 . & Chen, M.S. & UWA \\
\hline & & & 482. & Komarek, A.M. & USyd & 527 & Dharmaratna, D. & Monash \\
\hline & $\underline{2005}$ & & 483. & Lee, L.Y. & USyd & 528 & Gan, Q. & UNSW \\
\hline 437. & Ariffin, A. & Murdoch & 484. & Lester, L.H. & Flinders & 529 & Gray, E. & USyd \\
\hline 438. & Bel, R. & USyd & 485. & Martinez, L. & QUT & 530 & Hsu, P.K. & ANU \\
\hline 439. & Bianchi, R. & QUT & 486. & Poletti, S. & Auck & 531 & Jackson, A. & UNSW \\
\hline 440. & Cam, M.A. & RMIT & 487. & Roessler, C. & Melb & 532 & Ke, C. & Adel \\
\hline 441. & Chalil, D. & USyd & 488. & Skladzien, T. & Adel & 533. & Koska, O. & Otago \\
\hline 442. & Coate, B. & RMIT & 489. & Syed, I. & UNSW & 534 & Kostenko, W. & Monash \\
\hline 443. & Deodhar, V. & MacQ & 490. & Vo, D.H. & UWA & 535 & Lou, W. & $\mathrm{LTb}$ \\
\hline 444. & Doole, G. & UWA & 491. & Weatherston, C.R. & Otago & 536 & Mavisakalyan, A. & USyd \\
\hline 445. & Forrester, D. & UNSW & 492. & Worner, S. & ANU & 537. & Mendolia, S. & UNSW \\
\hline 446. & Ho, С.H. & UWA & 493. & Yang, S.S. & USyd & 538 & Moslehi, N. & UQ \\
\hline 447. & Hoque, S. & Monash & & & & 539 & Nencyz-Thiel, M. & USA \\
\hline 448. & Johnston, D. & Melb & & $\underline{2007}$ & & 540. & Nguyen, H.T. & ANU \\
\hline 449. & Kee, H.J. & ANU & 494. & Akhtar, $\overline{\text { S. }}$ & ANU & 541 & Nowak, S. & ANU \\
\hline 450. & Lee, W.S. & Melb & 495. & Alsaid, A. & UWA & 542 & Pham, H.Q. & ANU \\
\hline 451. & Leroux, A. & C'bridge & 496. & Barnes, T. & USyd & 543 & Rohde, N. & UQ \\
\hline 452. & Limkriangkrai, M. & UWA & 497. & Bunting, D. & UNSW & 544 & Schaffner, M. & QUT \\
\hline 453. & Liu, D.C. & UWA & 498. & Chen, Y. & Melb & 545 & Taborda, R. & ANU \\
\hline 454. & Olsen, T.S. & Monash & 499. & Djumashev, R. & Monash & 546 & Terdpaopong, K. & UNE \\
\hline 455. & Pacheco, G. & Auck & 500. & Farida, $\mathrm{M}$. & USyd & 547 & Tian, J. & ANU \\
\hline
\end{tabular}


TABLE A4 (continued)

THE STUDENTS, 1987-2019

\begin{tabular}{|c|c|c|c|c|c|c|c|c|}
\hline & Name & Affiliation & & Name & Affiliation & & Name & Affiliation \\
\hline 548. & Trott, D. & ANU & 593. & Chen, B. & UNSW & 640. & Nazifi, F. & MacQ \\
\hline 549. & Uylangco, K. & N'cast & 594. & Curtin, H. C. L. & Curtin & 641. & Nguyen, H. V. & UNSW \\
\hline 550. & Vehbi, M.T. & C'bridge & 595. & de Braganca, G. F. & VU.W'ton & 642. & Perera, S. & UNE \\
\hline 551. & Yamazaki, S. & ANU & 596. & Deakin, H. L. & Deakin & 643. & Pinto, P. & UQ \\
\hline 552. & Zhang, D. & ANU & 597. & Doan, M. & RMIT & 644. & Rai, A. & UNSW \\
\hline & 2009 & & $\begin{array}{l}598 \\
599\end{array}$ & $\begin{array}{l}\text { Edillon, R. } \\
\text { Fu, D. }\end{array}$ & $\begin{array}{l}\text { LTb } \\
\text { UWA }\end{array}$ & $\begin{array}{l}645 . \\
646 .\end{array}$ & $\begin{array}{l}\text { Shahiduzzaman, M. } \\
\text { Sharpe, T. }\end{array}$ & $\begin{array}{l}\text { USQ } \\
\text { N'cast }\end{array}$ \\
\hline 553. & Agarwal, M. & USA & 600 & Hassan, G. & UWS & 647. & Suziedelyte, A. & UNSW \\
\hline 554. & Andrienko, Y. & USyd & 601. & Hodge, A. & UQ & 648. & Wang, B. & MacQ \\
\hline 555. & Arora, V. & ANU & 602 & McCaughey, N. & Monash & 649. & Wang, C. & UNSW \\
\hline 556. & Athukorala, W. & QUT & 603 & McCracken, S. & ANU & 650. & Wang, J. L. & ANU \\
\hline 557. & Cai, Y. & ANU & 604 & McNair, B. & ANU & 651. & Xiang, V. & Monash \\
\hline 558. & Camacho, F. & UQ & 605 & Mujcic, R. & UQ & 652. & Yoo, H. I. & UNSW \\
\hline 559. & Carrington, S. & Monash & 606 & Ng, G. & Melb & 653. & $\mathrm{Yu}, \mathrm{F}$. & UWA \\
\hline 560. & Chow, V. S. S. & Melb & 607 & Ng, J. & Monash & 654. & Zhao, G. & ANU \\
\hline 561. & Etheridge, D. & UWA & 608 & Nguyen, X. & UNSW & & & \\
\hline 562. & Evans, S. & ANU & 609 & Palmer, M. & ANU & & $\underline{2012}$ & \\
\hline 563. & Gao, G. & UWA & 610 & Pavlov, O. & Adel & 655. & Al-Eitan, G. & Curtin \\
\hline 564. & Godfrey, K. & UWA & 611 & Pham, T. P. & USyd & 656. & Ansari, M. T. & QUT \\
\hline 565. & Gu, Y. & UNSW & 612 & Rai, A. & UNSW & 657. & Arsana, W. & UWA \\
\hline 566. & Hassan, AFM K. & Curtin & 613 & Sanderson, $\mathrm{T}$. & USyd & 658. & Chang, Y. & UTS \\
\hline 567. & Humberstone, R. & Melb & 614 & Shi, S. & ANU & 659. & Clarke, R. & UWA \\
\hline 568. & Inchauspe, J. & MacQ & 615 & van Gool, K. & UTS & 660. & Collins, J. & UWA \\
\hline 569. & Kim, N.-H. & Adel & 616 & Wells, R. & UWS & 661. & Ding, J. & MacQ \\
\hline 570. & Levy, A. & UNSW & 617 & Yan, E. & Monash & 662. & Dumrongrittikul, T. & Monash \\
\hline 571. & Lim, J. K. H. & UNSW & 618 & Zhang, W. & Melb & 663. & Gharibnavaz, M. R. & $\mathrm{LTb}$ \\
\hline 572. & Liu, S. & N'cast & & & & 664. & Khan, M. I. & $\mathrm{LTb}$ \\
\hline 573. & Lounkaew, K. & ANU & & $\underline{2011}$ & & 665. & Khezr, P. & USyd \\
\hline 574. & Marsden, A. & UTas & 619 & Ahmed, S. & Monash & 666. & Liu, Z. & UQ \\
\hline 575. & McCartney, A. & UWA & 620 & Bakshi, R. & Deakin & 667. & $\mathrm{Lu}, \mathrm{Y}$. & ANU \\
\hline 576. & Mohd, I. H. & Flinders & 621 & Besnek, M. & $\mathrm{LTb}$ & 668. & Marais, C. & Melb \\
\hline 577. & Nguyen, T. P. & Grif & 622 & Chowdhury, M. & Deakin & 669. & Mukherjee, R. & UWS \\
\hline 578. & Nicholas, A. & Monash & 623 & Feng, J. & UNSW & 670. & Nguyen, L. & Monash \\
\hline 579. & Paichayontvijit, T. & Auck & 624 & Fooken, J. & QUT & 671. & Norman, R. & UTS \\
\hline 580. & Shi, L. & UTS & 625 & Gounder, N. & Grif & 672. & Pakrashi, D. & UQ \\
\hline 581. & Shu, Y. & USyd & 626. & Guo, J. & Deakin & 673. & Sahgal, S. & UNSW \\
\hline 582. & Suh, J. Y. & UTS & 627. & Halstead, I. & Melb & 674. & Wang, C. & UNSW \\
\hline 583. & Suryadarma, D. & ANU & 628 & Hanna, D. & ANU & 675. & Wang, D. & Monash \\
\hline 584. & Taylor, W. & VU.W'ton & 629 & Hasan, Z. & UWS & 676. & Wang, G. J. & Melb \\
\hline 585. & Timol, I. & Monash & 630 & Hililan, M. & W'gong & 677. & Wang, J. & USyd \\
\hline 586. & Verani, S. & UCSB & 631. & Jiang, N. & Auck & 678. & Wang, X. & Auck \\
\hline 587. & Zhu, Y. & ANU & 632 & Lee, M. & UTS & 679. & Wiriyawit, V. & ANU \\
\hline & & & 633. & $\mathrm{Li}, \mathrm{L}$. & UWA & 680. & Wong, L. & Grif \\
\hline & 2010 & & 634 & $\mathrm{Li}, \mathrm{Q}$. & Auck & 681. & Yao, Y. & Melb \\
\hline 588. & Abdullaev, B. & LTb & 635 & Liu, Y. & USyd & 682. & Yong, S. K. & UQ \\
\hline 589. & Arshad, M. N. M. & UTas & 636 & Loch, A. & USA & 683. & Yu, C.-F. & Monash \\
\hline 590. & Bohlmann, H. & Monash & 637 & Maneesoonthorn, O. & Monash & 684. & Zheng, J. & ANU \\
\hline 591. & Brooke, G. & Auck & 638 & Marawila, T. D. & USyd & & & \\
\hline 592. & Che, X. G. & USyd & 639 & Montecinos, J. & UQ & & & \\
\hline
\end{tabular}


TABLE A4 (continued)

THE STUDENTS, 1987-2019

\begin{tabular}{|c|c|c|c|c|c|c|c|c|}
\hline & Name & Affiliation & & Name & Affiliation & & Name & Affiliation \\
\hline & $\underline{2013}$ & & 731. & Okabe, $\mathrm{T}$. & ANU & 776. & Goryacheva, E. & UTS \\
\hline 685. & Ambrey, C. & Grif & 732. & Phan, K. & USyd & 777. & Gunasinghe, C. & Grif \\
\hline 686. & Askarov, Z. & Deakin & 733. & Sami, J. & N'cast & 778. & Han, J. & UQ \\
\hline 687. & Cheng, T. & Monash & 734. & Shafiullah, M. & Grif & 779. & Hartigan, L. & UNSW \\
\hline 688. & Chinzara, Z. & QUT & 735. & Wijerathna, D. & Grif & 780. & Hou, C. & ANU \\
\hline 689. & Doan, D. & ANU & 736 . & $\mathrm{Wu}, \mathrm{K}$. & Monash & 781. & Johnson, S. & ANU \\
\hline 690. & Flavel, J. & Flinders & 737. & $\mathrm{Xu}, \mathrm{X}$. & UNSW & 782. & Kettlewell, N. & UNSW \\
\hline 691. & Hall, J. & UNSW & 738 & Xue, S. & ANU & 783. & Khuu, J. & Curtin \\
\hline 692. & Helal, M. & Melb & 739 & Ye, L. & UWA & 784. & Leung, F. & USyd \\
\hline 693. & Hoang, N. & $\mathrm{LTb}$ & 740 & Zheng, X. & UQ & 785. & Leung, P. & Monash \\
\hline 694. & Hsiao, C. & ANU & 741. & Zhu, H. & Monash & 786. & Liu, H. & UWA \\
\hline 695. & Humphreys, J. & UQ & & & & 787. & Ma, Q. & ANU \\
\hline 696. & Iles, R. & Grif & & $\underline{2015}$ & & 788. & Mangadi, K. & MacQ \\
\hline 697. & Joarder, M. & Curtin & 742 & Allen, $\overline{\mathrm{D} .}$ & RMIT & 789. & Nguyen, S. T. & UWA \\
\hline 698. & Lee, P. & USyd & 743 & Avcioglu, S. & Auck & 790. & Pan, G. & ANU \\
\hline 699. & Liyanaarachchi, $\mathrm{T}$. & Grif & 744 & Baikenova, Z. & Deakin & 791. & Sayeed, M. A. & UTas \\
\hline 700. & Okatch, Z. & UWA & 745 & Chen, L. & Monash & 792. & So, $\mathrm{T}$. & Auck \\
\hline 701. & Sabet, A. & UWA & 746 & Gauriot, R. & QUT & 793. & Swami, M. & Melb \\
\hline 702. & Savage, D. & UQ & 747 & Jayasinghe, M. & Grif & 794. & Swami, N. & Melb \\
\hline 703. & Schroder, M. & ANU & 748 & Jiang, D. & Adel & 795. & Trinh, K. & UQ \\
\hline 704. & Sinclair, S. & RMIT & 749 & Koh, W. C. & ANU & 796. & Ye, Y. & USyd \\
\hline 705. & So, C. & Monash & 750 & Kolluru, S. & MacQ & 797. & Zhang, E. J. & UNSW \\
\hline 706. & Tong, E. & Auck & 751. & La Nauze, A. & Melb & & & \\
\hline 707. & Triandaru, S. & MacQ & 752 & Neal, T. & UNSW & & $\underline{2017}$ & \\
\hline 708. & Ward, S. & Melb & 753 & Nguyen, C. & Monash & 798. & Amarasinghe, A. & Monash \\
\hline 709. & Wei, W. & ANU & 754 & Pan, A. & Massey & 799. & Blanco, A. & Melb \\
\hline 710. & Xiao, S. & UTS & 755 & Pereira, J. & UQ & 800. & Chen, Y. & UTS \\
\hline 711. & Xue, S. & ANU & 756 & Pham, H. T. & UQ & 801. & Chernulich, A. & UTS \\
\hline 712. & Yap, N. & UTS & 757 & Pham, V. & UNSW & 802. & Deij, S. & Monash \\
\hline 713. & Yuan, D. & Adel & 758 & Ravinthirakumaran, K. & Grif & 803. & Descamps, A. & QUT \\
\hline 714. & Zhou, Y. & ANU & 759 & Shi, X. & UQ & 804. & Drummond, P. & ANU \\
\hline & & & 760 & Singh, R. & Curtin & 805. & Hayek, D. & UNSW \\
\hline & $\underline{2014}$ & & 761. & Trinh, K. & UQ & 806. & Hendrawan, A. & ANU \\
\hline 715. & Andric, $\overline{S .}$ & Monash & 762 & Uzeda Garcia, L. & ANU & 807. & Hoelzemann, J. & UNSW \\
\hline 716. & Bowers, C. & MacQ & 763. & Vecci, J. & Monash & 808. & Hu, X. & RMIT \\
\hline 717. & Brugler, J. & C'bridge & 764. & Yeo, T. & UQ & 809. & Huang, J. & Melb \\
\hline 718. & Burfurd, I. & Melb & 765 & Zhu, L. & USyd & 810. & Kalsi, J. & Curtin \\
\hline 719. & Chen, A. & Auck & & & & 811. & Koh, B. & Melb \\
\hline 720. & Hasanov, R. & Deakin & & $\underline{2016}$ & & 812. & Kong, X. & USyd \\
\hline 721. & Huynh, L. & UQ & 766. & Balogh, A. & USyd & 813. & Leng, A. & UQ \\
\hline 722. & Hyder, Z. & ANU & 767 & Cai, P. & USyd & 814. & Li, Y. & Auck \\
\hline 723. & Jones, K. & Monash & 768 & Chen, Q. & Monash & 815. & Liu, C. & UNSW \\
\hline 724. & Khan, M. & Curtin & 769 & Coffie, R. O. & UWA & 816. & Liu, Z. & Monash \\
\hline 725. & Liu, W. & ANU & 770 & Dai, Y. & Adel & 817. & Loaiza, R. & MBS \\
\hline 726. & Liu, Y. & UNSW & 771. & Delacrétaz, D. & Melb & 818. & Mai, V. & Deakin \\
\hline 727. & Mamaghani, Y. T. & UWA & 772 & Ding, D. & UWA & 819. & Moncayo, D. & ANU \\
\hline 728. & McKay, M. & ANU & 773 & Dorner, Z. & Monash & 820. & Morris, T. & Melb \\
\hline 729. & Murray, C. & UQ & 774 & Garrard, R. & Adel & 821. & Nguyen, H. & Monash \\
\hline 730. & Nidup, J. & RMIT & 775 & Gholami, M. B. & Auck & 822. & Pham, D. & UQ \\
\hline
\end{tabular}


TABLE A4 (continued)

THE STUDENTS, 1987-2019

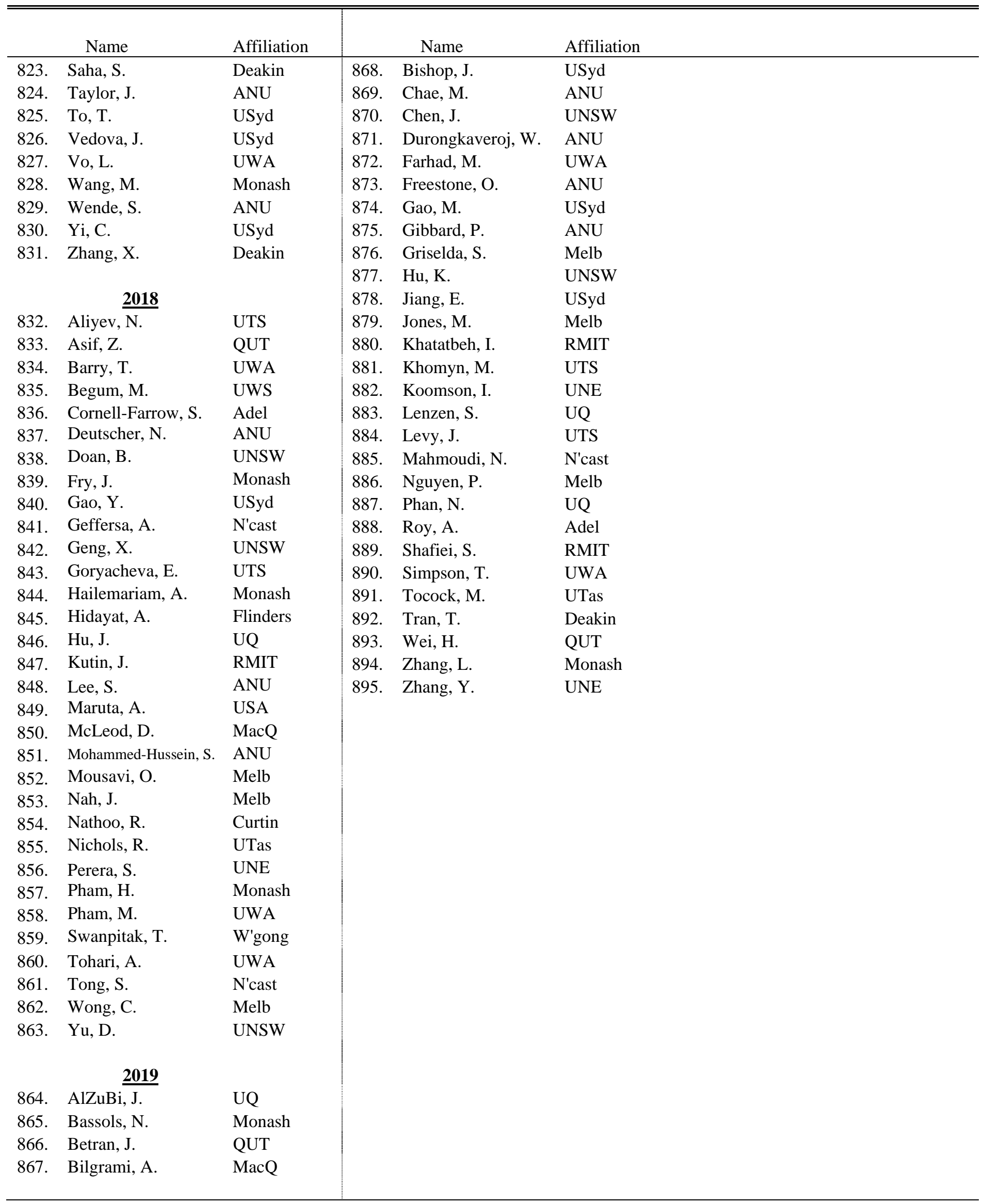

